\title{
Optimal Shape of Non-Linear Partially Wet Annular Fins for Maximum Efficiency
}

\author{
Cheng-Hung Huang * and Yun-Lung Chung
}

check for updates

Citation: Huang, C.-H.; Chung, Y.-L. Optimal Shape of Non-Linear Partially Wet Annular Fins for Maximum Efficiency. Energies 2021, 14, 844. https://doi.org/10.3390/ en14040844

Academic Editor: Jose

A. Almendros-lbanez

Received: 28 December 2020

Accepted: 2 February 2021

Published: 5 February 2021

Publisher's Note: MDPI stays neutral with regard to jurisdictional claims in published maps and institutional affiliations.

Copyright: (c) 2021 by the authors. Licensee MDPI, Basel, Switzerland. This article is an open access article distributed under the terms and conditions of the Creative Commons Attribution (CC BY) license (https:// creativecommons.org/licenses/by/ $4.0 /)$.

\author{
Department of Systems and Naval Mechatronic Engineering, National Cheng Kung University, Tainan 701, \\ Taiwan; verumasa0517@gmail.com \\ * Correspondence: chhuang@mail.ncku.edu.tw
}

\begin{abstract}
Heat exchangers with annular finned-tube type and partially wetted condition are utilized widely in engineering systems, such as air-conditioning systems and refrigeration systems. In addition, the physical properties of fin materials should be considered as functions of temperature in reality and thus become a non-linear problem. Based on the above two conditions, an optimal partially wet annular fin design problem, with temperature-dependent thermal properties of the fin, to yield optimal fin efficiency was investigated in the present work, which has not been examined previously and it is the novelty of this study. An iterative regularization algorithm using the conjugate gradient method (CGM) is considered as the optimization tool based on the desired fin efficiency under a fixed fin volume constraint. The partially wet annular fin condition can result if the relative humidity of surrounding air is between 80 and $90 \%$. Finally, the optimal fin shape, with the highest computed efficiency among examined fins under identical operational conditions, can be obtained. It is found that when the Biot numbers for ambient air $\left(\mathrm{Bi}_{\mathrm{a}}\right)$ and relative humidity $(\phi)$ increased, the optimum computed fin efficiency and interfacial radius between wet and dry fin domains $\left(\mathrm{r}_{\mathrm{wd}}\right)$ will be increased, and the estimated optimum fin shape also changed. However, the shape of optimal fin remained approximately unchanged when the Biot numbers for the inner tube $\left(\mathrm{Bi}_{\mathrm{i}}\right)$, the thermal conductivities of the tube $\left(\mathrm{k}_{\mathrm{w}}\right)$ and fin $\left(\mathrm{k}_{\mathrm{f}}\right)$ varied. It reveals that $\mathrm{Bi}_{\mathrm{i}}, \mathrm{k}_{\mathrm{W}}$ and $\mathrm{k}_{\mathrm{f}}$ have an insignificant influence on the optimal shape of the annular fin in a partially wet condition.
\end{abstract}

Keywords: partially wet annular fins; non-linear design problem; conjugate gradient method

\section{Introduction}

Depending on the dew point of the surrounding air, fin base temperature, and boundary temperature, the fin surface conditions can be classified as dry, partially wet, or fully wet. For the case of condensers, the fin surface temperature is generally below the dew point temperature; as a result, heat and mass transfer occur simultaneously, and the fin surface is partially wetted. In addition, the physical properties of fin and tube materials are considered functions of temperature, in reality, thus, the investigation of non-linear design problem becomes important. Therefore an optimal partially wet annular fin design problem with temperature-dependent physical properties of fin materials is investigated in this study and the fin efficiency can thus be improved.

Many researchers have investigated the heat and mass transfer behaviors of fin-andtube heat exchangers under dehumidifying conditions and have determined the temperature distribution and efficiency of fins. For instance, Charters and Theerakulpisut [1] examined the efficiency equations for constant-thickness annular fins. They have derived a simplified relationship to compute the fin efficiency of a specified annular fin. Mokheime [2] studied the performance of different annular fins with a locally variable heat transfer coefficient, the results were presented in a series of fin-efficiency curves for different annular fin shapes with a wide range of radius ratios. Iborra and Campo [3] investigated the temperature variation and efficiency for annular fins with a constant fin thickness. The fin efficiency of a one-dimensional fully wet rectangular longitudinal fin 
was determined by McQuiston [4]. Toner et al. [5] utilized a quasi-linear model to analyze one-dimensional fully wet rectangular and triangular fins. A criterion for optimum shape was proposed by Schmidt [6] using the principle of constant heat flux. Kern and Kraus [7] reported the investigation of the optimum design of wetted fin surfaces.

Recently, Jang et al. [8] investigated experimentally the enhancement of the heat transfer rate of steam condensation on the external surfaces of a vertical tube with annular fins. The applicability of finned tubes to a large condenser system with a bundle layout was verified. Xu et al. [9] computed the heat transfer capacity and air-side pressure drop of an annular radiator (AR) using a combined heat transfer unit (HTU) simulation and plate-and-fin heat exchanger (PFHX) performance calculation formulas. Results indicated that their optimization method could provide significant guidance for AR design.

An analytical expression of the efficiency for a longitudinal straight fin under dry, fully wet, and partially wet surface conditions was first reported by Wu and Bong [10]. They concluded that only when the fin is partially wet does the overall fin efficiency strongly depend on the relative humidity. The heat transfer characteristics of an annular fin under dry, partially wet, and fully wet surface conditions were studied by Naphon [11]; the results obtained from the study were validated by comparing them with those obtained by other researchers.

The "finite circular fin method" (FCFM) was utilized by Pirompugd et al. [12] to analyze the performance of fin-and-tube heat exchangers with a plain fin configuration under dehumidifying conditions. The heat and mass performance for the plain fin configuration under fully wet and partially wet conditions was described by the proposed correlations. Rosario and Rahman [13] presented a heat transfer analysis in a partially wet annular fin assembly during dehumidification. It was concluded in their work that the heat transfer performance can be improved significantly under condensation conditions.

Numerous linear fin shape design problems were investigated to optimize the annular fin profiles with constant thermal properties. For example, a variation separation method was used by Kang [14] in determining the fin length of a fully wet annular rectangular fin to achieve optimal fin efficiency. They have concluded that when assuming constant thermal conductivity, the optimum length and effectiveness of fins are independent of the fin material, however, the optimum base thickness and the volume of the fin are inversely proportional to the fin conductivity. The optimal shapes of uniform fully wet annular fins were examined by Brown [15]. A comparison was made between the optimal dimensions of the uniform fins and the dimensions of fins with minimum weight.

In the above reviewed references, the optimal fin shapes were estimated based on the criterion of minimizing the weight of fin or maximizing fin base heat transfer rate, with constant thermal properties condition. Fin shape design problems based on the desired fin efficiency under a fixed fin volume constraint are very limited in the literature.

Huang and Hsiao [16] utilized an iteration regularization algorithm (Alifanov [17]) in a fin design problem to estimate the optimal shapes for the spine and longitudinal fins based on the desired fin efficiency and a fixed fin volume. Huang and Chung investigated non-linear dry [18] and fully wet [19] fin design problems to determine the optimal shapes for the annular fins using the CGM based on the desired fin efficiency and a fixed fin volume constraint.

A fin design algorithm with temperature-dependent physical properties of annular fins using the CGM was considered in this work. It is an extension work of Huang and Chung [18,19], and the aim was to determine the optimal shapes of partially wet annular fins based on the desired fin efficiency under a fixed fin volume constraint which has not been investigated previously.

\section{The Direct Problem}

The heat and mass transfer equations, together with the boundary conditions, of a linear, steady-state partially wet annular fin adhered to a bare tube were defined by Huang and Chung [20]. If the thermal conductivity of fin and tube and the convective heat transfer 
coefficient are assumed as a function of temperatures, the governing equation of the present study can be obtained as:

Energy equation in tube wall:

$$
\frac{1}{\overline{\mathrm{r}}} \frac{\mathrm{d}}{\mathrm{d} \overline{\mathrm{r}}}\left[\overline{\mathrm{r}}_{\mathrm{w}}\left(\overline{\mathrm{T}}_{\mathrm{w}}\right) \frac{\mathrm{d} \overline{\mathrm{T}}_{\mathrm{w}}(\overline{\mathrm{r}})}{\mathrm{d} \overline{\mathrm{r}}}\right]=0 ; \text { in } \overline{\mathrm{r}}_{\mathrm{b}} \leq \overline{\mathrm{r}} \leq \overline{\mathrm{r}}_{\mathrm{o}}
$$

Energy equation in wet fin:

$$
\begin{gathered}
\frac{d}{d \bar{r}}\left[\overline{\mathrm{k}}_{\mathrm{f}}\left(\overline{\mathrm{T}}_{\mathrm{f}, \mathrm{w}}\right) \overline{\mathrm{A}}(\overline{\mathrm{r}}) \frac{\mathrm{d} \overline{\mathrm{T}}_{\mathrm{f}, \mathrm{w}}(\overline{\mathrm{r}})}{\mathrm{d} \overline{\mathrm{r}}}\right]=\overline{\mathrm{h}}_{\mathrm{o}}\left(\overline{\mathrm{T}}_{\mathrm{f}, \mathrm{w}}\right) \overline{\mathrm{S}}(\overline{\mathrm{r}})\left\{\left[\overline{\mathrm{T}}_{\mathrm{f}, \mathrm{w}}(\overline{\mathrm{r}})-\overline{\mathrm{T}}_{\mathrm{h}}\right]\right. \\
\left.+\frac{\overline{\mathrm{h}}_{\mathrm{f}} \overline{\mathrm{h}}_{\mathrm{d}}}{\overline{\mathrm{h}}_{\mathrm{o}}\left(\overline{\mathrm{T}}_{\mathrm{f}}\right)}\left[\bar{\omega}_{\mathrm{f}}\left(\overline{\mathrm{T}}_{\mathrm{f}, \mathrm{w}}\right)-\bar{\omega}_{\mathrm{h}}\right]\right\} ; \text { in } \overline{\mathrm{r}}_{\mathrm{o}} \leq \overline{\mathrm{r}} \leq \overline{\mathrm{r}}_{\mathrm{wd}}
\end{gathered}
$$

Energy equation in dry fin:

$$
\frac{\mathrm{d}}{\mathrm{d} \overline{\mathrm{r}}}\left[\overline{\mathrm{k}}_{\mathrm{f}}\left(\overline{\mathrm{T}}_{\mathrm{f}, \mathrm{d}}\right) \overline{\mathrm{A}}(\overline{\mathrm{r}}) \frac{\mathrm{d} \overline{\mathrm{T}}_{\mathrm{f}, \mathrm{d}}(\overline{\mathrm{r}})}{\mathrm{d} \overline{\mathrm{r}}}\right]=\overline{\mathrm{h}}_{\mathrm{o}}\left(\overline{\mathrm{T}}_{\mathrm{f}, \mathrm{d}}\right) \overline{\mathrm{S}}(\overline{\mathrm{r}})\left[\overline{\mathrm{T}}_{\mathrm{f}, \mathrm{d}}(\overline{\mathrm{r}})-\overline{\mathrm{T}}_{\mathrm{h}}\right] ; \text { in } \overline{\mathrm{r}}_{\mathrm{wd}} \leq \overline{\mathrm{r}} \leq \overline{\mathrm{r}}_{\mathrm{t}}
$$

Boundary condition on inner wall surface:

$$
-\overline{\mathrm{k}}_{\mathrm{w}}\left(\overline{\mathrm{T}}_{\mathrm{w}}\right) \frac{\mathrm{d} \overline{\mathrm{T}}_{\mathrm{w}}}{\mathrm{d} \overline{\mathrm{r}}}=\overline{\mathrm{h}}_{\mathrm{b}}\left(\overline{\mathrm{T}}_{\mathrm{w}}\right)\left(\overline{\mathrm{T}}_{\mathrm{c}}-\overline{\mathrm{T}}_{\mathrm{w}}\right) ; \text { at } \overline{\mathrm{r}}=\overline{\mathrm{r}}_{\mathrm{b}}
$$

Boundary condition at tube wall and wet fin interface:

$$
\begin{aligned}
& \overline{\mathrm{T}}_{\mathrm{w}}=\overline{\mathrm{T}}_{\mathrm{f}, \mathrm{w}} ; \text { at } \overline{\mathrm{r}}=\overline{\mathrm{r}}_{\mathrm{o}} \\
& -\overline{\mathrm{k}}_{\mathrm{w}}\left(\overline{\mathrm{T}}_{\mathrm{w}}\right) \overline{\mathrm{P}} \frac{\mathrm{d} \overline{\mathrm{T}}_{\mathrm{w}}}{\mathrm{d} \overline{\mathrm{r}}}=\overline{\mathrm{h}}_{\mathrm{o}}\left(\overline{\mathrm{T}}_{\mathrm{f}, \mathrm{w}}\right)\left(\overline{\mathrm{P}}-\frac{1}{2} \bar{\delta}\right)\left\{\left(\overline{\mathrm{T}}_{\mathrm{w}}-\overline{\mathrm{T}}_{\mathrm{h}}\right)+\frac{\overline{\mathrm{h}}_{\mathrm{f}} \overline{\mathrm{h}}_{\mathrm{d}}}{\overline{\mathrm{h}}_{\mathrm{o}}\left(\overline{\mathrm{T}}_{\mathrm{f}}\right)}\left[\bar{\omega}_{\mathrm{f}}\left(\overline{\mathrm{T}}_{\mathrm{f}, \mathrm{w}}\right)-\bar{\omega}_{\mathrm{h}}\right]\right\} \\
& -\frac{1}{2} \overline{\mathrm{k}}_{\mathrm{f}}\left(\overline{\mathrm{T}}_{\mathrm{f}, \mathrm{w}}\right) \bar{\delta} \frac{\mathrm{d} \overline{\mathrm{d}}_{\mathrm{f}, \mathrm{w}}}{\mathrm{d} \overline{\mathrm{r}}} ; \text { at } \overline{\mathrm{r}}=\overline{\mathrm{r}}_{\mathrm{o}}
\end{aligned}
$$

Boundary condition at wet and dry fins interface:

$$
\begin{aligned}
\overline{\mathrm{T}}_{\mathrm{f}, \mathrm{w}} & =\overline{\mathrm{T}}_{\mathrm{f}, \mathrm{d}} ; \text { at } \overline{\mathrm{r}}=\overline{\mathrm{r}}_{\mathrm{wd}} \\
\frac{\mathrm{d} \overline{\mathrm{T}}_{\mathrm{f}, \mathrm{w}}}{\mathrm{d} \overline{\mathrm{r}}} & =\frac{\mathrm{d} \overline{\mathrm{T}}_{\mathrm{f}, \mathrm{d}}}{\mathrm{d} \overline{\mathrm{r}}} ; \text { at } \overline{\mathrm{r}}=\overline{\mathrm{r}}_{\mathrm{wd}}
\end{aligned}
$$

Boundary condition at fin tip:

$$
-\overline{\mathrm{k}}_{\mathrm{f}}\left(\overline{\mathrm{T}}_{\mathrm{f}, \mathrm{d}}\right) \frac{\mathrm{d} \overline{\mathrm{T}}_{\mathrm{f}, \mathrm{d}}}{\mathrm{d} \overline{\mathrm{r}}}=\overline{\mathrm{h}}_{\mathrm{o}}\left(\overline{\mathrm{T}}_{\mathrm{f}, \mathrm{d}}\right)\left(\overline{\mathrm{T}}_{\mathrm{f}, \mathrm{d}}-\overline{\mathrm{T}}_{\mathrm{h}}\right) ; \text { at } \overline{\mathrm{r}}=\overline{\mathrm{r}}_{\mathrm{t}}
$$

where " - " indicates the dimensional variables and $\overline{\mathrm{T}}_{\mathrm{f}}, \overline{\mathrm{T}}_{\mathrm{W}}, \overline{\mathrm{T}}_{\mathrm{c}}$ and $\overline{\mathrm{T}}_{\mathrm{h}}$ are the temperatures for fin, tube, refrigerant and air, respectively.

The specific humidity of air on the fin surface can be calculated with $\bar{\omega}_{\mathrm{f}}\left(\overline{\mathrm{T}}_{\mathrm{f}}\right)=\mathrm{a}_{2}+\mathrm{b}_{2} \overline{\mathrm{T}}$ the values of $a_{2}$ and $b_{2}$ depend on the relative humidity and can be obtained in accordance with the equations reported by Sharqawy and Zubair [21]. In the present study, $\overline{\mathrm{T}}_{\mathrm{h}}$ and $\overline{\mathrm{T}}_{\mathrm{c}}$ are considered as $20^{\circ} \mathrm{C}$ and $2{ }^{\circ} \mathrm{C}$, respectively, and $\overline{\mathrm{r}}_{\mathrm{b}}, \overline{\mathrm{r}}_{\mathrm{o}}$ and $\overline{\mathrm{r}}_{\mathrm{t}}$ are considered to be 30,60 and $150 \mathrm{~mm}$, respectively. $\overline{\mathrm{r}}_{\mathrm{wd}}$ indicates the interface radius of wet and dry fin regions, it can be obtained iteratively until the interfacial condition (1g) is satisfied. The relative humidity $\phi$ from 0.8 to 0.85 and then 0.9 is selected to ensure that the dew point will occur within the annular fin and a partially wet annular fin condition will yield. The results of $\overline{\mathrm{A}}(\overline{\mathrm{r}})=2 \pi \overline{\mathrm{r}} \bar{\delta}$ and $\overline{\mathrm{S}}(\overline{\mathrm{r}})=4 \pi \overline{\mathrm{r}}$ are obtained by assuming that $\bar{\delta}(\overline{\mathrm{r}})<<\overline{\mathrm{S}}(\overline{\mathrm{r}})$. 
By defining the non-dimensionl parameters below:

$$
\begin{aligned}
& \theta_{\mathrm{W}}=\frac{\overline{\mathrm{T}}_{\mathrm{w}}-\overline{\mathrm{T}}_{\mathrm{h}}}{\overline{\mathrm{T}}_{\mathrm{c}}-\overline{\mathrm{T}}_{\mathrm{h}}} ; \theta_{\mathrm{f}, \mathrm{w}}=\frac{\overline{\mathrm{T}}_{\mathrm{f}, \mathrm{w}}-\overline{\mathrm{T}}_{\mathrm{h}}}{\overline{\mathrm{T}}_{\mathrm{c}}-\overline{\mathrm{T}}_{\mathrm{h}}} ; \theta_{\mathrm{f}, \mathrm{d}}=\frac{\overline{\mathrm{T}}_{\mathrm{f}, \mathrm{d}}-\overline{\mathrm{T}}_{\mathrm{h}}}{\overline{\mathrm{T}}_{\mathrm{c}}-\overline{\mathrm{T}}_{\mathrm{h}}} ; \mathrm{r}=\frac{\overline{\mathrm{r}}}{\overline{\mathrm{r}}_{\mathrm{t}}} ; \mathrm{P}=\frac{\overline{\mathrm{P}}}{\overline{\mathrm{T}_{\mathrm{t}}}} ; \delta=\frac{\bar{\delta}}{\overline{\mathrm{r}}_{\mathrm{t}}} \\
& \mathrm{k}_{\mathrm{w}}\left(\theta_{\mathrm{W}}\right)=\frac{\overline{\mathrm{k}}_{\mathrm{w}}\left(\bar{T}_{\mathrm{w}}\right)}{\overline{\mathrm{k}}_{\mathrm{r}}} ; \mathrm{k}_{\mathrm{f}}\left(\theta_{\mathrm{f}, \mathrm{W}}\right)=\frac{\overline{\mathrm{k}}_{\mathrm{f}}\left(\overline{\mathrm{T}}_{\mathrm{f}, \mathrm{w}}\right)}{\overline{\mathrm{k}}_{\mathrm{r}}} ; \mathrm{k}_{\mathrm{f}}\left(\theta_{\mathrm{f}, \mathrm{d}}\right)=\frac{\overline{\mathrm{k}}_{\mathrm{f}}\left(\overline{\mathrm{T}}_{\mathrm{f}, \mathrm{d}}\right)}{\overline{\mathrm{k}}_{\mathrm{r}}} \\
& \operatorname{Bi}_{\mathrm{i}}\left(\theta_{\mathrm{w}}\right)=\frac{\overline{\mathrm{h}}_{\mathrm{b}}\left(\overline{\mathrm{T}}_{\mathrm{w}}\right) \overline{\mathrm{r}}_{\mathrm{t}}}{\overline{\mathrm{k}}_{\mathrm{r}}} ; \mathrm{Bi}_{\mathrm{a}}\left(\theta_{\mathrm{f}, \mathrm{w}}\right)=\frac{\overline{\mathrm{h}}_{\mathrm{o}}\left(\overline{\mathrm{T}}_{\mathrm{f}, \mathrm{w}}\right) \overline{\mathrm{r}}_{\mathrm{t}}}{\overline{\mathrm{k}}_{\mathrm{r}}} ; \mathrm{Bi}_{\mathrm{a}}\left(\theta_{\mathrm{f}, \mathrm{d}}\right)=\frac{\overline{\mathrm{h}}_{\mathrm{o}}\left(\overline{\mathrm{T}}_{\mathrm{f}, \mathrm{d}}\right) \overline{\mathrm{r}}_{\mathrm{t}}}{\overline{\mathrm{k}}_{\mathrm{r}}}
\end{aligned}
$$

The following dimensionless equations for partially wet fin equations and boundary conditions are obtained:

$$
\begin{aligned}
& \frac{1}{\mathrm{r}} \frac{\mathrm{d}}{\mathrm{dr}}\left[\mathrm{rk}_{\mathrm{w}}\left(\theta_{\mathrm{w}}\right) \frac{\mathrm{d} \theta_{\mathrm{w}}(\mathrm{r})}{\mathrm{dr}}\right]=0 ; \text { in } \mathrm{r}_{\mathrm{b}} \leq \mathrm{r} \leq \mathrm{r}_{\mathrm{o}} \\
& \frac{1}{\mathrm{r}} \frac{\mathrm{d}}{\mathrm{dr}}\left[\mathrm{rk}_{\mathrm{f}}\left(\theta_{\mathrm{f}, \mathrm{w}}\right) \delta(\mathrm{r}) \frac{\mathrm{d} \theta_{\mathrm{f}, \mathrm{w}}(\mathrm{r})}{\mathrm{dr}}\right] \\
& =2 \mathrm{Bi}_{\mathrm{a}}\left(\theta_{\mathrm{f}, \mathrm{w}}\right)\left\{\theta_{\mathrm{f}, \mathrm{w}}(\mathrm{r})+\overline{\mathrm{h}}_{\mathrm{fg}} \frac{\left(\mathrm{C}_{\mathrm{p}, \mathrm{a}} \mathrm{L}_{\mathrm{e}}{ }^{2 / 3}\right)^{-1}}{\left(\overline{\mathrm{T}}_{\mathrm{c}}-\overline{\mathrm{T}}_{\mathrm{h}}\right)}\left[\bar{\omega}_{\mathrm{f}}\left(\overline{\mathrm{T}}_{\mathrm{f}, \mathrm{w}}\right)-\bar{\omega}_{\mathrm{h}}\right]\right\} ; \text { in } \mathrm{r}_{\mathrm{o}} \leq \mathrm{r} \leq \mathrm{r}_{\mathrm{wd}} \\
& \frac{1}{\mathrm{r}} \frac{\mathrm{d}}{\mathrm{dr}}\left[\mathrm{rk}_{\mathrm{f}}\left(\theta_{\mathrm{f}, \mathrm{d}}\right) \delta(\mathrm{r}) \frac{\mathrm{d} \theta_{\mathrm{f}, \mathrm{d}}(\mathrm{r})}{\mathrm{dr}}\right]=2 \mathrm{Bi}_{\mathrm{a}}\left(\theta_{\mathrm{f}, \mathrm{d}}\right) \theta_{\mathrm{f}, \mathrm{d}}(\mathrm{r}) ; \text { in } \mathrm{r}_{\mathrm{wd}} \leq \mathrm{r} \leq 1 \\
& -\mathrm{k}_{\mathrm{w}}\left(\theta_{\mathrm{w}}\right) \frac{\mathrm{d} \theta_{\mathrm{w}}}{\mathrm{dr}}=\mathrm{Bi}_{\mathrm{i}}\left(\theta_{\mathrm{w}}\right)\left(1-\theta_{\mathrm{w}}\right) ; \text { at } \mathrm{r}=\mathrm{r}_{\mathrm{b}} \\
& \theta_{\mathrm{w}}=\theta_{\mathrm{f}, \mathrm{w}} ; \text { at } \mathrm{r}=\mathrm{r}_{\mathrm{o}} \\
& -\mathrm{k}_{\mathrm{w}}\left(\theta_{\mathrm{w}}\right) \mathrm{P} \frac{\mathrm{d} \theta_{\mathrm{w}}}{\mathrm{dr}}=\left(\mathrm{P}-\frac{1}{2} \delta\right) \mathrm{Bi}_{\mathrm{a}}\left(\theta_{\mathrm{f}, \mathrm{w}}\right)\left\{\theta_{\mathrm{w}}+\overline{\mathrm{h}}_{\mathrm{fg}} \frac{\left(\mathrm{C}_{\mathrm{p}, \mathrm{a}} \mathrm{L}_{\mathrm{e}}^{2 / 3}\right)^{-1}}{\left(\overline{\mathrm{T}}_{\mathrm{c}}-\overline{\mathrm{T}}_{\mathrm{h}}\right)}\left[\bar{\omega}_{\mathrm{f}}\left(\overline{\mathrm{T}}_{\mathrm{f}, \mathrm{w}}\right)-\bar{\omega}_{\mathrm{h}}\right]\right\} \\
& -\frac{1}{2} \mathrm{k}_{\mathrm{f}}\left(\theta_{\mathrm{f}, \mathrm{w}}\right) \delta \frac{\mathrm{d} \theta_{\mathrm{f}, \mathrm{w}}}{\mathrm{dr}} ; \text { at } \mathrm{r}=\mathrm{r}_{\mathrm{o}} \\
& \theta_{\mathrm{f}, \mathrm{w}}=\theta_{\mathrm{f}, \mathrm{d}} ; \text { at } \mathrm{r}=\mathrm{r}_{\mathrm{wd}} \\
& \frac{d \theta_{f, w}}{d r}=\frac{d \theta_{f, d}}{d r} ; \text { at } r=r_{w d} \\
& -\mathrm{k}_{\mathrm{f}}\left(\theta_{\mathrm{f}, \mathrm{d}}\right) \frac{\mathrm{d} \theta_{\mathrm{f}, \mathrm{d}}}{\mathrm{dr}}=\mathrm{Bi}_{\mathrm{a}}\left(\theta_{\mathrm{f}, \mathrm{d}}\right) \theta_{\mathrm{f}, \mathrm{d}} ; \text { at } \mathrm{r}=1
\end{aligned}
$$

The identity $\frac{h_{d}}{\bar{h}_{o}\left(\bar{T}_{f}\right)}=\left(C_{p, a} L_{e}{ }^{2 / 3}\right)^{-1}[22]$ is adopted and $L_{e}=S_{c} / P_{r}=0.864$. The geometries of a 3-D and 2-D partially wet annular fins are given in Figure 1a,b respectively. The direct problem considered above is to determine the temperature distribution and efficiency of a non-linear partially wet fin when the fin shape is provided. Once dimensionless temperatures $\theta$ are calculated, the dimensional temperatures $\overline{\mathrm{T}}$ can be obtained by utilizing dimensionless quantities $\theta_{\mathrm{w}}=\frac{\overline{\mathrm{T}}_{\mathrm{w}}-\overline{\mathrm{T}}_{\mathrm{h}}}{\overline{\mathrm{T}}_{\mathrm{c}}-\overline{\mathrm{T}}_{\mathrm{h}}}, \theta_{\mathrm{f}, \mathrm{w}}=\frac{\overline{\mathrm{T}}_{\mathrm{f}, \mathrm{w}}-\overline{\mathrm{T}}_{\mathrm{h}}}{\overline{\mathrm{T}}_{\mathrm{c}}-\overline{\mathrm{T}}_{\mathrm{h}}}$ and $\theta_{\mathrm{f}, \mathrm{d}}=\frac{\overline{\mathrm{T}}_{\mathrm{f}, \mathrm{d}}-\overline{\mathrm{T}}_{\mathrm{h}}}{\overline{\mathrm{T}}_{\mathrm{c}}-\overline{\mathrm{T}}_{\mathrm{h}}}$ with $\overline{\mathrm{T}}_{\mathrm{h}}=20^{\circ} \mathrm{C}$ and $\overline{\mathrm{T}}_{\mathrm{C}}=2{ }^{\circ} \mathrm{C}$. 


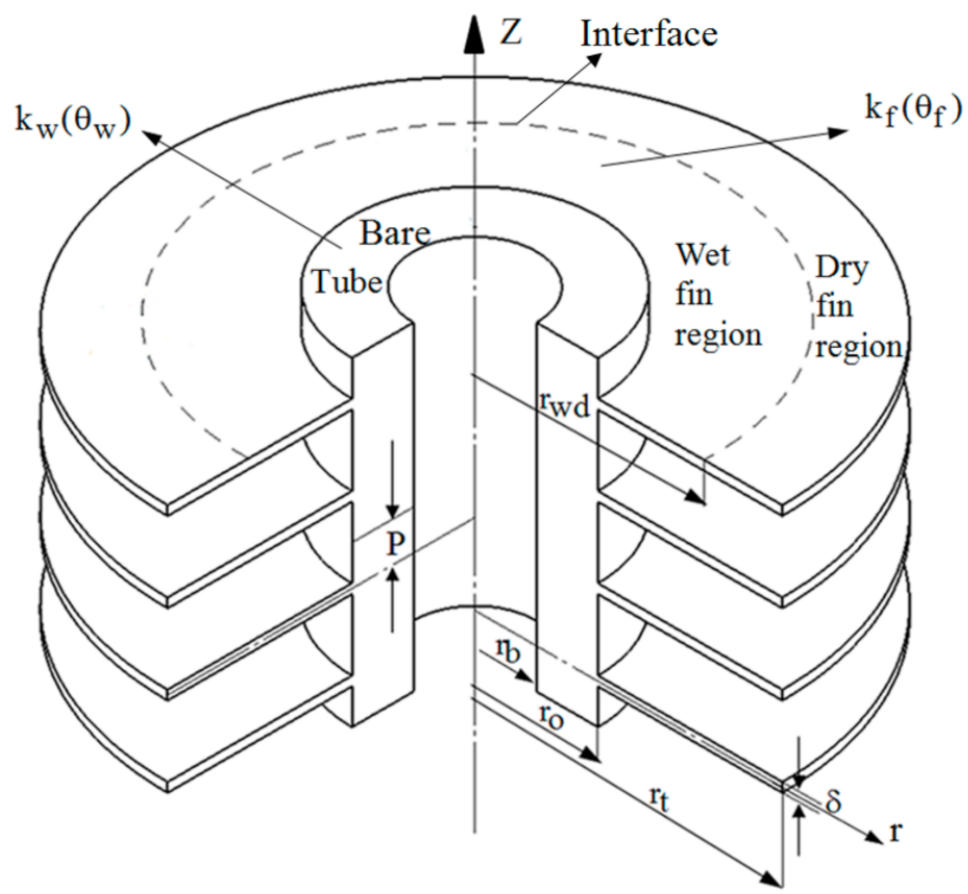

(a)

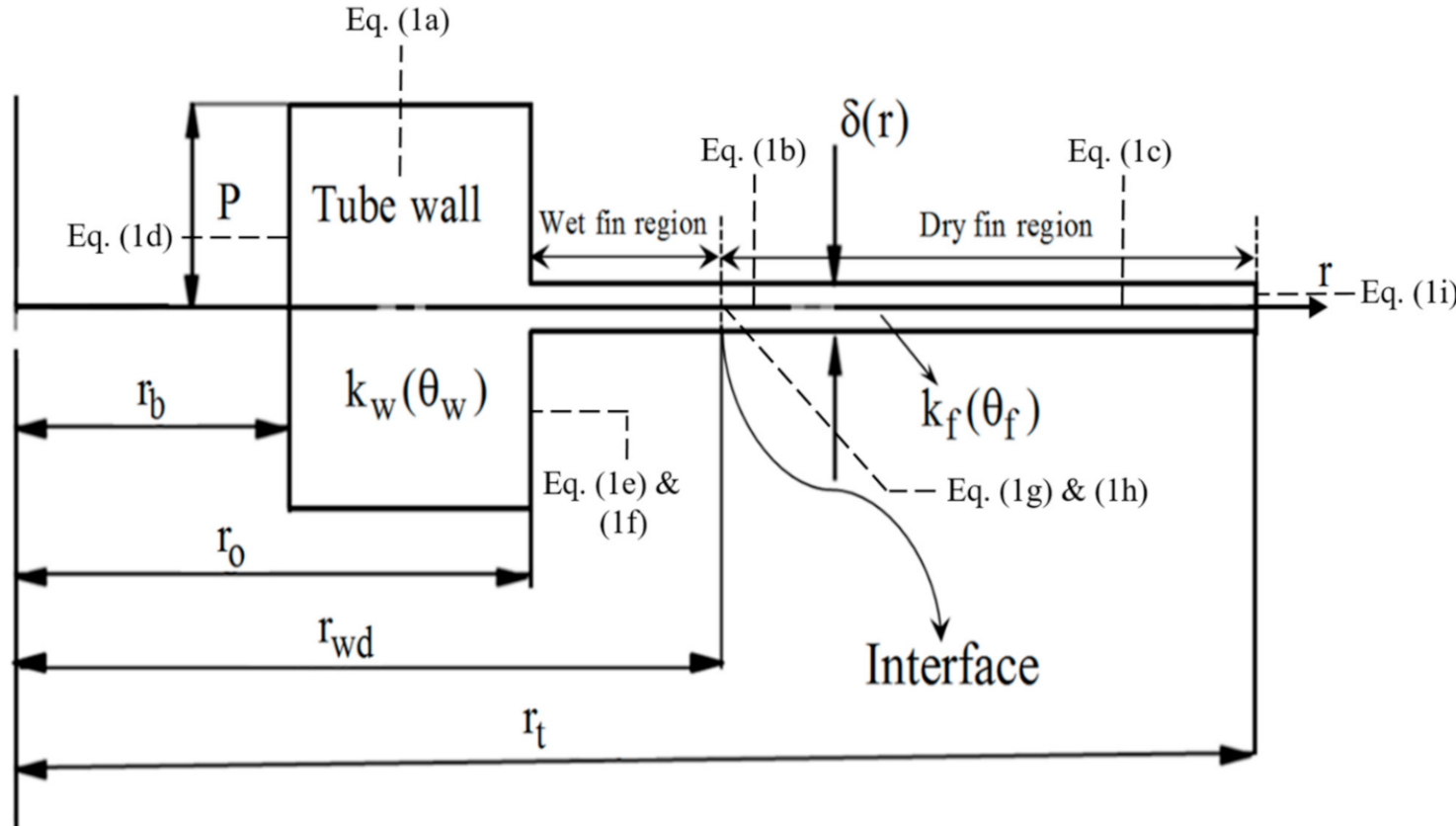

(b)

Figure 1. (a) A three-dimensional geometry of partially wet annular fins. (b) A cross-sectional view of a single annular fin.

\section{The Fin Design Problem}

An inverse algorithm to estimate the optimal shapes based on the desired fin efficiency under a fixed fin volume constraint is discussed.

The following cost function $\mathrm{J}(\delta)$ needs to be minimized with respect to $\delta(\mathrm{r})$ to yield the optimal shape of the annular fin in this study under the design criterion of desired fin efficiency and fin volume:

$$
J(\delta)=[q(\delta)-\Phi Q(\delta)]^{2}+\alpha[v(\delta)-V]^{2}
$$


Here $\Phi$ (or $\eta)=q / Q$ is defined as the desired (or estimated) fin efficiency, and the estimated heat transfer rate $\mathrm{q}(\delta)$ represents heat transfer rate in reality, while $Q(\delta)$ indicates the heat transfer rate obtained by assuming that fin surface temperature equal to fin base temperature $\bar{T}_{0}$, i.e., the ideal heat transfer rate. $v(\delta)$ and $V$ are the computed and desired fin volumes, respectively, and $\alpha$ denotes the weighting coefficient.

The iterative process should be stopped if the value of the cost function, $J(\delta)$, is smaller than the stopping condition, $\varepsilon$, and the optimal shape of the partially wet fin can be obtained.

The following new variables, $\mathrm{q}=\frac{\overline{\mathrm{q}}}{\overline{\mathrm{q}}_{\text {ref }}}, \mathrm{Q}=\frac{\overline{\mathrm{Q}}}{\overline{\mathrm{q}}_{\text {ref }}}$ and $\mathrm{v}=\frac{\overline{\mathrm{v}}}{\overline{\mathrm{V}}_{\text {ref }}}$ need be defined, and the reference quantities are given below:

$$
\begin{gathered}
\overline{\mathrm{q}}_{\text {ref }}=\overline{\mathrm{k}}_{\mathrm{r}} \overline{\mathrm{r}}_{\mathrm{t}}\left(\overline{\mathrm{T}}_{\mathrm{c}}-\overline{\mathrm{T}}_{\mathrm{h}}\right) \\
\overline{\mathrm{v}}_{\text {ref }}=\overline{\mathrm{r}}_{\mathrm{t}}^{3}
\end{gathered}
$$

For annular fins, the equations below can be derived:

$$
\begin{aligned}
\overline{\mathrm{q}} & =\int_{\overline{\mathrm{r}}=\overline{\mathrm{r}}_{\mathrm{o}}}^{\overline{\mathrm{r}}_{\mathrm{wd}}} \overline{\mathrm{h}}_{\mathrm{o}}\left(\overline{\mathrm{T}}_{\mathrm{f}, \mathrm{w}}\right)(4 \pi \overline{\mathrm{r}})\left\{\left(\overline{\mathrm{T}}_{\mathrm{f}, \mathrm{w}}-\overline{\mathrm{T}}_{\mathrm{h}}\right)+\overline{\mathrm{h}}_{\mathrm{fg}}\left(\mathrm{C}_{\mathrm{p}, \mathrm{a}} \mathrm{L}_{\mathrm{e}}^{2 / 3}\right)^{-1}\left[\overline{\mathrm{w}}_{\mathrm{f}}\left(\overline{\mathrm{T}}_{\mathrm{f}, \mathrm{w}}\right)-\overline{\boldsymbol{\omega}}_{\mathrm{h}}\right]\right\} \mathrm{d} \overline{\mathrm{r}} \\
& +\int_{\overline{\mathrm{r}}=\overline{\mathrm{r}}_{\mathrm{wd}}}^{\overline{\mathrm{r}}_{\mathrm{f}}} \overline{\mathrm{h}}_{\mathrm{o}}\left(\overline{\mathrm{T}}_{\mathrm{f}, \mathrm{d}}\right)(4 \pi \overline{\mathrm{r}})\left(\overline{\mathrm{T}}_{\mathrm{f}, \mathrm{d}}-\overline{\mathrm{T}}_{\mathrm{h}}\right) \mathrm{d} \overline{\mathrm{r}}
\end{aligned}
$$

The conditions $\overline{\mathrm{T}}_{\mathrm{f}, \mathrm{w}}=\overline{\mathrm{T}}_{\mathrm{f}, \mathrm{d}}=\overline{\mathrm{T}}_{\mathrm{o}}=$ constant are utilized in Equation (5a) to compute $\overline{\mathrm{Q}}$ :

$$
\begin{gathered}
\overline{\mathrm{Q}}=\overline{\mathrm{h}}_{\mathrm{o}}\left(\overline{\mathrm{T}}_{\mathrm{o}}\right)\left\{\left(\overline{\mathrm{T}}_{\mathrm{o}}-\overline{\mathrm{T}}_{\mathrm{h}}\right)+\overline{\mathrm{h}}_{\mathrm{fg}}\left(\mathrm{C}_{\mathrm{p}, \mathrm{a}} \mathrm{L}_{\mathrm{e}}{ }^{2 / 3}\right)^{-1}\left[\bar{\omega}_{\mathrm{f}}\left(\overline{\mathrm{T}}_{\mathrm{o}}\right)-\bar{\omega}_{\mathrm{h}}\right]\right\} \int_{\overline{\mathrm{r}}=\overline{\mathrm{r}}_{\mathrm{o}}}^{\overline{\mathrm{r}}_{\mathrm{wd}}}(4 \pi \overline{\mathrm{r}}) \mathrm{d} \overline{\mathrm{r}} \\
+\overline{\mathrm{h}}_{\mathrm{o}}\left(\overline{\mathrm{T}}_{\mathrm{o}}\right)\left(\overline{\mathrm{T}}_{\mathrm{o}}-\overline{\mathrm{T}}_{\mathrm{h}}\right) \int_{\overline{\mathrm{r}}=\overline{\mathrm{r}}_{\mathrm{wd}}}^{\overline{\mathrm{r}}_{\mathrm{t}}}(4 \pi \overline{\mathrm{r}}) \mathrm{d} \overline{\mathrm{r}} \\
\overline{\mathrm{v}}=\int_{\overline{\mathrm{r}}=\overline{\mathrm{r}}_{\mathrm{o}}}^{\overline{\mathrm{r}}_{\mathrm{t}}} 2 \pi \bar{\delta} \overline{\mathrm{r}} \mathrm{d} \overline{\mathrm{r}}
\end{gathered}
$$

The expressions of $\mathrm{q}, \mathrm{Q}$ and $\mathrm{v}$ in dimensionless form are given below:

$$
\begin{aligned}
& \mathrm{q}=\int_{\mathrm{r}=\mathrm{r}_{\mathrm{o}}}^{\mathrm{r}_{\mathrm{wd}}} 4 \pi \mathrm{rBi} \mathrm{i}_{\mathrm{a}}\left(\theta_{\mathrm{f}, \mathrm{w}}\right)\left\{\theta_{\mathrm{f}, \mathrm{w}}(\mathrm{r})+\overline{\mathrm{h}}_{\mathrm{fg}}\left(\mathrm{C}_{\mathrm{p}, \mathrm{a}} \mathrm{L}_{\mathrm{e}}{ }^{2 / 3}\right)^{-1} \frac{\left[\overline{\mathrm{w}}_{\mathrm{f}}\left(\overline{\mathrm{T}}_{\mathrm{f}, \mathrm{w}}\right)-\overline{\mathrm{w}}_{\mathrm{h}}\right]}{\left(\overline{\mathrm{T}}_{\mathrm{c}}-\overline{\mathrm{T}}_{\mathrm{h}}\right)}\right\} \mathrm{dr} \\
& +\int_{\mathrm{r}=\mathrm{r}_{\mathrm{wd}}}^{1} 4 \pi \mathrm{rBi}_{\mathrm{a}}\left(\theta_{\mathrm{f}, \mathrm{d}}\right) \theta_{\mathrm{f}, \mathrm{d}}(\mathrm{r}) \mathrm{dr} \\
& \mathrm{Q}=\mathrm{Bi}_{\mathrm{a}}\left(\theta_{\mathrm{o}}\right)\left\{\theta_{\mathrm{o}}+\overline{\mathrm{h}}_{\mathrm{fg}}\left(\mathrm{C}_{\mathrm{p}, \mathrm{a}} \mathrm{L}_{\mathrm{e}}{ }^{2 / 3}\right)^{-1} \frac{\left[\bar{\omega}_{\mathrm{f}}\left(\overline{\mathrm{T}}_{\mathrm{o}}\right)-\overline{\boldsymbol{\omega}}_{\mathrm{h}}\right]}{\left(\overline{\mathrm{T}}_{\mathrm{c}}-\overline{\mathrm{T}}_{\mathrm{h}}\right)}\right\}_{\mathrm{r}=\mathrm{r}_{\mathrm{o}}}^{\mathrm{r}_{\mathrm{wd}}} 4 \pi \mathrm{rdr} \\
& +\mathrm{Bi}_{\mathrm{a}}\left(\theta_{\mathrm{o}}\right) \theta_{\mathrm{o}} \int_{\mathrm{r}=\mathrm{r}_{\mathrm{wd}}}^{1} 4 \pi \mathrm{rdr} \\
& \mathrm{v}=\int_{\mathrm{r}=\mathrm{r}_{\mathrm{o}}}^{1} 2 \pi \delta(\mathrm{r}) \mathrm{rdr}
\end{aligned}
$$

The present design problem of the non-linear partially wet annular fin can thus be expressed as: based on the cost function $\mathrm{J}(\delta)$, design the optimal partially wet fin shape, such that $\mathrm{q}$ approach to $\Phi \mathrm{Q}$ and $\mathrm{v}$ approach to V. 


\section{Iterative Process with CGM}

The optimal annular fin thickness, $\delta(\mathrm{r})$, can be obtained by minimizing the cost function, $J[\delta(r)]$, using the following CGM iteration equation (Alifanov [17]):

$$
\delta^{\mathrm{n}+1}(\mathrm{r})=\delta^{\mathrm{n}}(\mathrm{r})-\beta^{\mathrm{n}} \mathrm{p}^{\mathrm{n}}(\mathrm{r})
$$

where $\beta^{\mathrm{n}}$ and $\mathrm{p}^{\mathrm{n}}(\mathrm{r})$ denote the step size and search direction for each iteration. The expression of $\mathrm{p}^{\mathrm{n}}(\mathrm{r})$ is given below:

$$
\mathrm{p}^{\mathrm{n}}(\mathrm{r})=\mathrm{J}^{\mathrm{n}}(\mathrm{r})+\gamma^{\mathrm{n}} \mathrm{p}^{\mathrm{n}-1}(\mathrm{r})
$$

Here the conjugate coefficient $\gamma^{\mathrm{n}}$ is given as (Alifanov [17]):

$$
\gamma^{\mathrm{n}}=\int_{\mathrm{r}=\mathrm{r}_{\mathrm{o}}}^{1}\left(\mathrm{~J}^{\mathrm{n}}\right)^{2} \mathrm{dr} / \int_{\mathrm{r}=\mathrm{r}_{\mathrm{o}}}^{1}\left(\mathrm{~J}^{\mathrm{n}-1}\right)^{2} \mathrm{dr} ; \text { with } \gamma^{0}=0
$$

In order to perform the iterative process in accordance with Equation (6a), the values of $\beta^{n}$ and $J^{\prime n}[\delta(r)]$ must be calculated. The governing equations for sensitivity and adjoint problems must be derived since their solutions are used to calculate the values of $\beta^{\mathrm{n}}$ and $\mathrm{J}^{\prime \mathrm{n}}[\delta(\mathrm{r})]$.

\section{The Sensitivity Problem}

The sensitivity problem can be obtained by utilizing the perturbational principle, that is, when $\delta(\mathrm{r})$ has a perturbation $\Delta \delta(\mathrm{r})$, all the dependent variables will be varied by $\Delta \theta_{\mathrm{w}}(\mathrm{r}), \Delta \theta_{\mathrm{f}}(\mathrm{r}), \Delta \overline{\mathrm{w}}, \Delta \mathrm{k}_{\mathrm{w}}\left(\theta_{\mathrm{w}}\right), \Delta \mathrm{k}_{\mathrm{f}}\left(\theta_{\mathrm{f}}\right), \Delta \mathrm{Bi}_{\mathrm{i}}\left(\theta_{\mathrm{w}}\right)$, and $\Delta \mathrm{Bi}_{\mathrm{a}}\left(\theta_{\mathrm{f}}\right)$, respectively. Based on the derivation procedure by Huang and Chung $[18,19]$, the governing equations for the sensitivity problem can be obtained as:

$$
\frac{1}{\mathrm{r}} \frac{\mathrm{d}}{\mathrm{dr}}\left[\mathrm{r} \Delta \mathrm{k}_{\mathrm{w}}\left(\theta_{\mathrm{w}}\right) \frac{\mathrm{d} \theta_{\mathrm{w}}(\mathrm{r})}{\mathrm{dr}}+\mathrm{rk}_{\mathrm{w}}\left(\theta_{\mathrm{w}}\right) \frac{\mathrm{d} \Delta \theta_{\mathrm{w}}(\mathrm{r})}{\mathrm{dr}}\right]=0 ; \text { in } \mathrm{r}_{\mathrm{b}} \leq \mathrm{r} \leq \mathrm{r}_{\mathrm{o}}
$$

$\frac{1}{\mathrm{r}} \frac{\mathrm{d}}{\mathrm{dr}}\left[\mathrm{rk}_{\mathrm{f}}\left(\theta_{\mathrm{f}, \mathrm{W}}\right) \delta(\mathrm{r}) \frac{\mathrm{d} \Delta \theta_{\mathrm{f}, \mathrm{w}}(\mathrm{r})}{\mathrm{dr}}\right]+\frac{1}{\mathrm{r}} \frac{\mathrm{d}}{\mathrm{dr}}\left\{\left[\mathrm{r}_{\mathrm{r}} \mathrm{k}_{\mathrm{f}}\left(\theta_{\mathrm{f}, \mathrm{W}}\right) \delta(\mathrm{r})+\mathrm{rk}_{\mathrm{f}}\left(\theta_{\mathrm{f}, \mathrm{W}}\right) \Delta \delta(\mathrm{r})\right] \frac{\mathrm{d} \theta_{\mathrm{f}, \mathrm{w}}(\mathrm{r})}{\mathrm{dr}}\right\}$

$=2\left[\mathrm{Bi}_{\mathrm{a}}\left(\theta_{\mathrm{f}, \mathrm{w}}\right) \Delta \theta_{\mathrm{f}, \mathrm{w}}(\mathrm{r})+\Delta \mathrm{Bi}_{\mathrm{a}}\left(\theta_{\mathrm{f}, \mathrm{w}}\right) \theta_{\mathrm{f}, \mathrm{w}}(\mathrm{r})\right]$

$+2 \overline{\mathrm{h}}_{\mathrm{fg}} \frac{\left(\mathrm{C}_{\mathrm{p}, \mathrm{a}} \mathrm{L}_{\mathrm{e}}^{2 / 3}\right)^{-1}}{\left(\overline{\mathrm{T}}_{\mathrm{c}}-\overline{\mathrm{T}}_{\mathrm{h}}\right)}\left\{\mathrm{Bi}_{\mathrm{a}}\left(\theta_{\mathrm{f}, \mathrm{w}}\right) \Delta \bar{\omega}_{\mathrm{f}}\left(\overline{\mathrm{T}}_{\mathrm{f}, \mathrm{w}}\right)+\Delta \mathrm{Bi}_{\mathrm{a}}\left(\theta_{\mathrm{f}, \mathrm{w}}\right)\left[\bar{\omega}_{\mathrm{f}}\left(\overline{\mathrm{T}}_{\mathrm{f}, \mathrm{w}}\right)-\bar{\omega}_{\mathrm{h}}\right]\right\} ;$ in $\mathrm{r}_{\mathrm{o}} \leq \mathrm{r} \leq \mathrm{r}_{\mathrm{wd}}$

$$
\begin{aligned}
& \frac{1}{\mathrm{r}} \frac{\mathrm{d}}{\mathrm{dr}}\left[\mathrm{rk}_{\mathrm{f}}\left(\theta_{\mathrm{f}, \mathrm{d}}\right) \delta(\mathrm{r}) \frac{\mathrm{d} \Delta \theta_{\mathrm{f}, \mathrm{d}}(\mathrm{r})}{\mathrm{dr}}\right]+\frac{1}{\mathrm{r}} \frac{\mathrm{d}}{\mathrm{dr}}\left\{\left[\mathrm{r} \Delta \mathrm{k}_{\mathrm{f}}\left(\theta_{\mathrm{f}, \mathrm{d}}\right) \delta(\mathrm{r})+\mathrm{rk}_{\mathrm{f}}\left(\theta_{\mathrm{f}, \mathrm{d}}\right) \Delta \delta(\mathrm{r})\right] \frac{\mathrm{d} \Delta \theta_{\mathrm{f}, \mathrm{d}}(\mathrm{r})}{\mathrm{dr}}\right\} \\
& =2\left[\operatorname{Bi}_{\mathrm{a}}\left(\theta_{\mathrm{f}, \mathrm{d}}\right) \Delta \theta_{\mathrm{f}, \mathrm{d}}(\mathrm{r})+\Delta \mathrm{Bi}_{\mathrm{a}}\left(\theta_{\mathrm{f}, \mathrm{d}}\right) \theta_{\mathrm{f}, \mathrm{d}}(\mathrm{r})\right] ; \text { in } \mathrm{r}_{\mathrm{wd}} \leq \mathrm{r} \leq 1 \\
& \mathrm{k}_{\mathrm{w}}\left(\theta_{\mathrm{w}}\right) \frac{\mathrm{d} \Delta \theta_{\mathrm{w}}}{\mathrm{dr}}+\Delta \mathrm{k}_{\mathrm{w}}\left(\theta_{\mathrm{w}}\right) \frac{\mathrm{d} \theta_{\mathrm{w}}}{\mathrm{dr}}=\mathrm{Bi}_{\mathrm{i}}\left(\theta_{\mathrm{w}}\right) \Delta \theta_{\mathrm{w}}-\Delta \mathrm{Bi}_{\mathrm{i}}\left(\theta_{\mathrm{w}}\right)\left(1-\theta_{\mathrm{w}}\right) ; \text { at } \mathrm{r}=\mathrm{r}_{\mathrm{b}} \\
& \Delta \theta_{\mathrm{w}}=\Delta \theta_{\mathrm{f}, \mathrm{w}} ; \text { at } \mathrm{r}=\mathrm{r}_{\mathrm{o}} \\
& -\mathrm{k}_{\mathrm{w}}\left(\theta_{\mathrm{w}}\right) \mathrm{p} \frac{\mathrm{d} \Delta \theta_{\mathrm{w}}}{\mathrm{dr}}-\Delta \mathrm{k}_{\mathrm{W}}\left(\theta_{\mathrm{w}}\right) \mathrm{p} \frac{\mathrm{d} \theta_{\mathrm{w}}}{\mathrm{dr}} \\
& =\left[\left(\mathrm{P}-\frac{1}{2} \delta\right) \Delta \mathrm{Bi}_{\mathrm{a}}\left(\theta_{\mathrm{f}, \mathrm{w}}\right)+\left(-\frac{1}{2} \Delta \delta\right) \mathrm{Bi}_{\mathrm{a}}\left(\theta_{\mathrm{f}, \mathrm{w}}\right)\right]\left\{\theta_{\mathrm{w}}+\overline{\mathrm{h}}_{\mathrm{fg}} \frac{\left(\mathrm{C}_{\mathrm{p}, \mathrm{a}} \mathrm{L}_{\mathrm{e}}{ }^{2 / 3}\right)^{-1}}{\left(\overline{\mathrm{T}}_{\mathrm{c}}-\overline{\mathrm{T}}_{\mathrm{h}}\right)}\left[\overline{\boldsymbol{\omega}}_{\mathrm{f}}\left(\overline{\mathrm{T}}_{\mathrm{f}, \mathrm{w}}\right)-\bar{\omega}_{\mathrm{h}}\right]\right\} \\
& +\left(\mathrm{P}-\frac{1}{2} \delta\right) \mathrm{Bi}_{\mathrm{a}}\left(\theta_{\mathrm{f}, \mathrm{w}}\right)\left\{\Delta \theta_{\mathrm{w}}+\overline{\mathrm{h}}_{\mathrm{fg}} \frac{\left(\mathrm{C}_{\mathrm{p}, \mathrm{a}} \mathrm{L}_{\mathrm{e}} \mathrm{e}^{2 / 3}\right)^{-1}}{\left(\overline{\mathrm{T}}_{\mathrm{c}}-\overline{\mathrm{T}}_{\mathrm{h}}\right)} \frac{\mathrm{d} \bar{\omega}_{\mathrm{f}}\left(\overline{\mathrm{T}}_{\mathrm{f}, \mathrm{w}}\right)}{\mathrm{d} \theta_{\mathrm{f}, \mathrm{w}}} \Delta \theta_{\mathrm{f}, \mathrm{w}}\right\} \\
& +\left\{-\frac{1}{2} \mathrm{k}_{\mathrm{f}}\left(\theta_{\mathrm{f}, \mathrm{W}}\right) \Delta \delta \frac{\mathrm{d} \theta_{\mathrm{f}, \mathrm{w}}}{\mathrm{dr}}-\frac{1}{2} \Delta \mathrm{k}_{\mathrm{f}}\left(\theta_{\mathrm{f}, \mathrm{W}}\right) \delta \frac{\mathrm{d} \theta_{\mathrm{f}, \mathrm{w}}}{\mathrm{dr}}-\frac{1}{2} \mathrm{k}_{\mathrm{f}}\left(\theta_{\mathrm{f}, \mathrm{w}}\right) \delta \frac{\mathrm{d} \Delta \theta_{\mathrm{f}, \mathrm{w}}}{\mathrm{dr}}\right\} \text { at } \mathrm{r}=\mathrm{r}_{\mathrm{o}} \\
& \Delta \theta_{\mathrm{f}, \mathrm{w}}=\Delta \theta_{\mathrm{f}, \mathrm{d}} ; \text { at } \mathrm{r}=\mathrm{r}_{\mathrm{wd}} \\
& \frac{\mathrm{d} \Delta \theta_{\mathrm{f}, \mathrm{w}}}{\mathrm{dr}}=\frac{\mathrm{d} \Delta \theta_{\mathrm{f}, \mathrm{d}}}{\mathrm{dr}} ; \text { at } \mathrm{r}=\mathrm{r}_{\mathrm{wd}}
\end{aligned}
$$




$$
-\left[\mathrm{k}_{\mathrm{f}}\left(\theta_{\mathrm{f}, \mathrm{d}}\right) \frac{\mathrm{d} \Delta \theta_{\mathrm{f}, \mathrm{d}}}{\mathrm{dr}}+\Delta \mathrm{k}_{\mathrm{f}}\left(\theta_{\mathrm{f}, \mathrm{d}}\right) \frac{\mathrm{d} \theta_{\mathrm{f}, \mathrm{d}}}{\mathrm{dr}}\right]=\mathrm{Bi}_{\mathrm{a}}\left(\theta_{\mathrm{f}, \mathrm{d}}\right) \Delta \theta_{\mathrm{f}, \mathrm{d}}+\Delta \mathrm{Bi}_{\mathrm{a}}\left(\theta_{\mathrm{f}, \mathrm{d}}\right) \theta_{\mathrm{f}, \mathrm{d}} ; \text { at } \mathrm{r}=1
$$

The cost function $\mathrm{J}\left(\delta^{\mathrm{n}+1}\right)$ can be obtained as:

$$
\begin{gathered}
\mathrm{J}\left(\delta^{\mathrm{n}+1}\right)=\left\{\mathrm{q}\left(\delta^{\mathrm{n}}\right)-\beta^{\mathrm{n}}\left[\Delta \mathrm{q}\left(\mathrm{p}^{\mathrm{n}}\right)-\Phi \Delta \mathrm{Q}\left(\mathrm{p}^{\mathrm{n}}\right)\right]-\Phi \mathrm{Q}\left(\delta^{\mathrm{n}}\right)\right\}^{2} \\
+\alpha\left[\mathrm{v}\left(\delta^{\mathrm{n}}\right)-\beta^{\mathrm{n}} \Delta \mathrm{v}\left(\mathrm{p}^{\mathrm{n}}\right)-\mathrm{V}\right]^{2}
\end{gathered}
$$

Equations for $\Delta \mathrm{q}, \Delta \mathrm{Q}$ and $\Delta \mathrm{v}$ can be derived utilizing the identities $\Delta \mathrm{q}=\mathrm{q}(\delta+\Delta \delta)-$ $\mathrm{q}(\delta), \Delta \mathrm{Q}=\mathrm{Q}(\delta+\Delta \delta)-\mathrm{Q}(\delta)$ and $\Delta \mathrm{v}=\mathrm{v}(\delta+\Delta \delta)-\mathrm{v}(\delta)$ and neglecting the higher order terms. Finally we have:

$$
\begin{aligned}
& \Delta \mathrm{q}=\int_{\mathrm{r}=\mathrm{r}_{\mathrm{o}}}^{\mathrm{r}_{\mathrm{wd}}} 4 \pi \mathrm{r}\left\{\mathrm{Bi}_{\mathrm{a}}\left(\theta_{\mathrm{f}, \mathrm{w}}\right)\left[\Delta \theta_{\mathrm{f}, \mathrm{w}}(\mathrm{r})+\overline{\mathrm{h}}_{\mathrm{fg}}\left(\mathrm{C}_{\mathrm{p}, \mathrm{a}} \mathrm{L}_{\mathrm{e}}^{2 / 3}\right)^{-1} \frac{\Delta \bar{\omega}_{\mathrm{f}}\left(\overline{\mathrm{T}}_{\mathrm{f}, \mathrm{w}}\right)}{\left(\overline{\mathrm{T}}_{\mathrm{c}}-\mathrm{T}_{\mathrm{h}}\right)}\right]\right. \\
& \left.+\Delta \mathrm{Bi}_{\mathrm{a}}\left(\theta_{\mathrm{f}, \mathrm{w}}\right)\left[\theta_{\mathrm{f}, \mathrm{w}}(\mathrm{r})+\overline{\mathrm{h}}_{\mathrm{fg}}\left(\mathrm{C}_{\mathrm{p}, \mathrm{a}} \mathrm{L}_{\mathrm{e}}{ }^{2 / 3}\right)^{-1} \frac{\bar{\omega}_{\mathrm{f}}\left(\overline{\mathrm{T}}_{\mathrm{f}, \mathrm{w}}\right)-\bar{\omega}_{\mathrm{h}}}{\left(\overline{\mathrm{T}}_{\mathrm{c}}-\overline{\mathrm{T}}_{\mathrm{h}}\right)}\right]\right\} \mathrm{dr} \\
& +\int_{r=r_{w d}}^{1} 4 \pi r\left[B_{a}\left(\theta_{f, d}\right) \Delta \theta_{f, d}(r)+\Delta \operatorname{Bi}_{a}\left(\theta_{f, d}\right) \theta_{f, d}(r)\right] d r \\
& \Delta \mathrm{Q}=\left\{\mathrm{Bi}_{\mathrm{a}}\left(\theta_{\mathrm{o}}\right)\left[\Delta \theta_{\mathrm{o}}+\overline{\mathrm{h}}_{\mathrm{fg}}\left(\mathrm{C}_{\mathrm{p}, \mathrm{a}} \mathrm{L}_{\mathrm{e}}{ }^{2 / 3}\right)^{-1} \frac{\Delta \bar{\omega}_{\mathrm{f}}\left(\overline{\mathrm{T}}_{\mathrm{o}}\right)}{\left(\overline{\mathrm{T}}_{\mathrm{c}}-\overline{\mathrm{T}}_{\mathrm{h}}\right)}\right]\right. \\
& \left.+\Delta \mathrm{Bi}_{\mathrm{a}}\left(\theta_{\mathrm{o}}\right)\left[\theta_{\mathrm{o}}+\overline{\mathrm{h}}_{\mathrm{fg}}\left(\mathrm{C}_{\mathrm{p}, \mathrm{a}} \mathrm{L}_{\mathrm{e}}{ }^{2 / 3}\right)^{-1} \frac{{\overline{\omega_{\mathrm{f}}}}_{\mathrm{f}}\left(\overline{\mathrm{T}}_{\mathrm{o}}\right)-\bar{\omega}_{\mathrm{h}}}{\left(\bar{T}_{\mathrm{c}}-\overline{\mathrm{T}}_{\mathrm{h}}\right)}\right]\right\} \int_{\mathrm{r}=\mathrm{r}_{\mathrm{o}}}^{\mathrm{r}_{\mathrm{wd}}} 4 \pi \mathrm{rdr} \\
& +\left[\operatorname{Bi}_{\mathrm{a}}\left(\theta_{\mathrm{o}}\right) \Delta \theta_{\mathrm{o}}+\Delta \mathrm{Bi}_{\mathrm{a}}\left(\theta_{\mathrm{o}}\right) \theta_{\mathrm{o}}\right] \int_{\mathrm{r}=\mathrm{r}_{\mathrm{wd}}}^{1} 4 \pi \mathrm{rdr} \\
& \Delta \mathrm{v}=\int_{\mathrm{r}=\mathrm{r}_{\mathrm{o}}}^{1} 2 \pi \Delta \delta(\mathrm{r}) \mathrm{rdr}
\end{aligned}
$$

The search step size $\beta^{\mathrm{n}}$ given below can be obtained by minimizing Equation ( $8 \mathrm{~b}$ ):

$$
\beta^{\mathrm{n}}=\frac{(\mathrm{q}-\Phi \mathrm{Q})(\Delta \mathrm{q}-\Phi \Delta \mathrm{Q})+\alpha(\mathrm{v}-\mathrm{V}) \Delta \mathrm{v}}{(\Delta \mathrm{q}-\Phi \Delta \mathrm{Q})^{2}+\alpha \Delta \mathrm{v}^{2}}
$$

\section{The Adjoint Problem}

The functions $\lambda_{\mathrm{w}}(\mathrm{r}), \lambda_{\mathrm{f}, \mathrm{W}}(\mathrm{r})$, and $\lambda_{\mathrm{f}, \mathrm{d}}(\mathrm{r})$ are multiplied to Equations (2a) and (2b), the resultant expressions are integrated over the space domain. The results are included in the RHS of Equation (3) to obtain the equation for $J(\delta)$ below:

$$
\begin{aligned}
& \mathrm{J}(\delta)=(\mathrm{q}-\Phi \mathrm{Q})^{2}+\alpha(\mathrm{v}-\mathrm{V})^{2} \\
& +\int_{\mathrm{r}=\mathrm{r}_{\mathrm{b}}}^{\mathrm{r}_{\mathrm{b}}} \lambda_{\mathrm{w}}(\mathrm{r}) \mathrm{r}\left\{\frac{1}{\mathrm{r}} \frac{\mathrm{d}}{\mathrm{dr}}\left[\mathrm{rk}_{\mathrm{w}}\left(\theta_{\mathrm{w}}\right) \frac{\mathrm{d} \theta_{w v}(\mathrm{r})}{\mathrm{dr}}\right]\right\} \mathrm{dr} \\
& +\int_{\mathrm{r}=\mathrm{r}_{\mathrm{o}}}^{\mathrm{r}_{\mathrm{wd}}} \lambda_{\mathrm{f}, \mathrm{w}}(\mathrm{r}) \mathrm{r}\left\{\frac{1}{\mathrm{r}} \frac{\mathrm{d}}{\mathrm{dr}}\left[\mathrm{rk}_{\mathrm{f}}\left(\theta_{\mathrm{f}, \mathrm{w}}\right) \delta(\mathrm{r}) \frac{\mathrm{d} \theta_{\mathrm{f}, \mathrm{w}}(\mathrm{r})}{\mathrm{dr}}\right]\right\} \mathrm{dr}+\int_{\mathrm{r}=\mathrm{r}_{\mathrm{wd}}}^{1} \lambda_{\mathrm{f}, \mathrm{d}}(\mathrm{r}) \mathrm{r}\left\{\frac{1}{\mathrm{r}} \frac{\mathrm{d}}{\mathrm{dr}}\left[\mathrm{rk}_{\mathrm{f}}\left(\theta_{\mathrm{f}, \mathrm{d}}\right) \delta(\mathrm{r}) \frac{\mathrm{d} \theta_{\mathrm{f}, \mathrm{d}}(\mathrm{r})}{\mathrm{dr}}\right]\right\} \mathrm{dr} \\
& -\int_{\mathrm{r}=\mathrm{r}_{\mathrm{o}}}^{\mathrm{r}_{\mathrm{w}}} \lambda_{\mathrm{f}, \mathrm{w}}(\mathrm{r}) \mathrm{r}\left[2 \mathrm{Bi}_{\mathrm{a}}\left(\theta_{\mathrm{f}, \mathrm{w}}\right)\right]\left\{\theta_{\mathrm{f}, \mathrm{w}}(\mathrm{r})+\overline{\mathrm{h}}_{\mathrm{fg}}\left(\mathrm{C}_{\mathrm{p}, \mathrm{a}} \mathrm{L}_{\mathrm{e}} \mathrm{e}^{2 / 3}\right)^{-1} \frac{\left[\overline{\mathrm{w}}_{\mathrm{f}}\left(\bar{T}_{\mathrm{f}}\right)-\overline{\mathrm{w}}_{\mathrm{h}}\right]}{\left(\mathrm{T}_{\mathrm{c}}-\overline{\mathrm{T}}_{\mathrm{h}}\right)}\right\} \mathrm{dr} \\
& -\int_{\mathrm{r}=\mathrm{r}_{\mathrm{wd}}}^{1} \lambda_{\mathrm{f}, \mathrm{d}}(\mathrm{r}) \mathrm{r}\left\{2 \mathrm{Bi}_{\mathrm{a}}\left(\theta_{\mathrm{f}, \mathrm{d}}\right) \theta_{\mathrm{f}, \mathrm{d}}(\mathrm{r})\right\}
\end{aligned}
$$

The perturbation, $\Delta \mathrm{J}$, can be obtained by following the standard procedure stated in $[18,19]$. Finally, it is given below: 


$$
\begin{aligned}
& \Delta \mathrm{J}=2(\mathrm{q}-\Phi \mathrm{Q})(\Delta \mathrm{q}-\Phi \Delta \mathrm{Q})+\alpha[2(\mathrm{v}-\mathrm{V}) \Delta \mathrm{v}] \\
& +\int_{\mathrm{r}=\mathrm{r}_{\mathrm{b}}}^{\mathrm{r}_{\mathrm{o}}} \lambda_{\mathrm{w}}(\mathrm{r}) \mathrm{r}\left\{\frac{1}{\mathrm{r}} \frac{\mathrm{d}}{\mathrm{dr}}\left[\mathrm{r} \Delta \mathrm{k}_{\mathrm{w}}\left(\theta_{\mathrm{w}}\right) \frac{\mathrm{d} \theta_{w}(\mathrm{r})}{\mathrm{dr}}+\mathrm{rk}_{\mathrm{w}}\left(\theta_{\mathrm{w}}\right) \frac{\mathrm{d} \Delta \theta_{w}(\mathrm{r})}{\mathrm{dr}}\right]\right\} \mathrm{dr} \\
& +\int_{\mathrm{r}=\mathrm{r}_{\mathrm{o}}}^{\mathrm{r}_{\mathrm{wd}}} \lambda_{\mathrm{f}, \mathrm{w}}(\mathrm{r}) \mathrm{r}\left\{\frac{1}{\mathrm{r}} \frac{\mathrm{d}}{\mathrm{dr}}\left[\mathrm{rk}_{\mathrm{f}}\left(\theta_{\mathrm{f}, \mathrm{w}}\right) \delta(\mathrm{r}) \frac{\mathrm{d} \Delta \theta_{\mathrm{f}, \mathrm{w}}(\mathrm{r})}{\mathrm{dr}}+\mathrm{r} \Delta \mathrm{k}_{\mathrm{f}}\left(\theta_{\mathrm{f}, \mathrm{w}}\right) \delta(\mathrm{r}) \frac{\mathrm{d} \theta_{\mathrm{f}, \mathrm{w}}(\mathrm{r})}{\mathrm{dr}}+\mathrm{rk}_{\mathrm{f}}\left(\theta_{\mathrm{f}, \mathrm{w}}\right) \Delta \delta(\mathrm{r}) \frac{\mathrm{d} \theta_{\mathrm{f}, \mathrm{w}}(\mathrm{r})}{\mathrm{dr}}\right]\right\} \mathrm{dr} \\
& +\int_{\mathrm{r}=\mathrm{r}_{\mathrm{wd}}}^{1} \lambda_{\mathrm{f}, \mathrm{d}}(\mathrm{r}) \mathrm{r}\left\{\frac{1}{\mathrm{r}} \frac{\mathrm{d}}{\mathrm{dr}}\left[\mathrm{rk}_{\mathrm{f}}\left(\theta_{\mathrm{f}, \mathrm{d}}\right) \delta(\mathrm{r}) \frac{\mathrm{d} \Delta \theta_{\mathrm{f}, \mathrm{d}}(\mathrm{r})}{\mathrm{dr}}+\mathrm{r} \Delta \mathrm{k}_{\mathrm{f}}\left(\theta_{\mathrm{f}, \mathrm{d}}\right) \delta(\mathrm{r}) \frac{\left.\mathrm{d}_{\mathrm{f}, \mathrm{d}} \mathrm{r}\right)}{\mathrm{dr}}+\mathrm{rk}_{\mathrm{f}}\left(\theta_{\mathrm{f}, \mathrm{d}}\right) \Delta \delta(\mathrm{r}) \frac{\mathrm{d} \theta_{\mathrm{f}, \mathrm{d}}(\mathrm{r})}{\mathrm{dr}}\right]\right\} \mathrm{dr} \\
& -\int_{\mathrm{r}=\mathrm{r}_{\mathrm{o}}}^{\mathrm{r}_{\mathrm{wd}}} \lambda_{\mathrm{f}, \mathrm{w}}(\mathrm{r}) \mathrm{r} 2\left[\mathrm{Bi}_{\mathrm{a}}\left(\theta_{\mathrm{f}, \mathrm{w}}\right) \Delta \theta_{\mathrm{f}, \mathrm{w}}(\mathrm{r})+\Delta \mathrm{Bi}_{\mathrm{a}}\left(\theta_{\mathrm{f}, \mathrm{w}}\right) \theta_{\mathrm{f}, \mathrm{w}}(\mathrm{r})\right] \mathrm{dr} \\
& -\int_{\mathrm{r}=\mathrm{r}_{\mathrm{o}}}^{\mathrm{r}_{\mathrm{wd}}} \lambda_{\mathrm{f}, \mathrm{w}}(\mathrm{r}) \mathrm{r} 2 \bar{h}_{\mathrm{fg}} \frac{\left(\mathrm{C}_{\mathrm{p}, \mathrm{a}} \mathrm{L}_{\mathrm{e}}{ }^{2 / 3}\right)}{\left(\overline{\mathrm{T}}_{\mathrm{c}}-\overline{\mathrm{T}}_{\mathrm{h}}\right)}\left\{\mathrm{Bi} \mathrm{i}_{\mathrm{a}}\left(\theta_{\mathrm{f}, \mathrm{w}}\right) \Delta \overline{\mathrm{w}}_{\mathrm{f}}\left(\overline{\mathrm{T}}_{\mathrm{f}, \mathrm{w}}\right)+\Delta \mathrm{Bi} \mathrm{i}_{\mathrm{a}}\left(\theta_{\mathrm{f}, \mathrm{w}}\right)\left[\bar{\omega}_{\mathrm{f}}\left(\overline{\mathrm{T}}_{\mathrm{f}, \mathrm{w}}\right)-\bar{\omega}_{\mathrm{h}}\right]\right\} \mathrm{dr} \\
& -\int_{\mathrm{r}=\mathrm{r}_{\mathrm{wd}}}^{1} \lambda_{\mathrm{f}, \mathrm{d}}(\mathrm{r}) \mathrm{r} 2\left[\mathrm{Bi}_{\mathrm{a}}\left(\theta_{\mathrm{f}, \mathrm{d}}\right) \Delta \theta_{\mathrm{f}, \mathrm{d}}(\mathrm{r})+\Delta \mathrm{Bi}_{\mathrm{a}}\left(\theta_{\mathrm{f}, \mathrm{d}}\right) \theta_{\mathrm{f}, \mathrm{d}}(\mathrm{r})\right] \mathrm{dr}
\end{aligned}
$$

The space integrations in Equation (12) are integrated by parts, Equations (7c)-(7f) are utilized, and then $\Delta \mathrm{J}$ is the approach to zero. The following governing equations for adjoint problem are obtained:

$$
\begin{aligned}
& \mathrm{k}_{\mathrm{w}}\left(\theta_{\mathrm{w}}\right) \frac{1}{\mathrm{r}} \frac{\mathrm{d}}{\mathrm{dr}}\left[\mathrm{r} \frac{\mathrm{d} \lambda_{\mathrm{w}}(\mathrm{r})}{\mathrm{dr}}\right]=0 ; \text { in } \mathrm{r}_{\mathrm{b}} \leq \mathrm{r} \leq \mathrm{r}_{\mathrm{o}} \\
& \mathrm{k}_{\mathrm{f}}\left(\theta_{\mathrm{f}, \mathrm{w}}\right) \frac{1}{\mathrm{r}} \frac{\mathrm{d}}{\mathrm{dr}}\left[\mathrm{r} \delta(\mathrm{r}) \frac{\mathrm{d} \lambda_{\mathrm{f}, \mathrm{w}}(\mathrm{r})}{\mathrm{dr}}\right]+8 \pi \mathrm{r}(\mathrm{q}-\Phi Q)\left\{\mathrm{Bi}_{\mathrm{a}}\left(\theta_{\mathrm{f}, \mathrm{w}}\right)+\frac{\mathrm{dBi}_{\mathrm{a}}\left(\theta_{\mathrm{f}, \mathrm{w}}\right)}{\mathrm{d} \theta_{\mathrm{f}, \mathrm{w}}(\mathrm{r})} \theta_{\mathrm{f}, \mathrm{w}}(\mathrm{r})\right\} \\
& +8 \pi r(q-\Phi Q) \bar{h}_{f g} \frac{\left(C_{p, a} L_{e} e^{2 / 3}\right)^{-1}}{\left(\bar{T}_{c}-\bar{T}_{h}\right)}\left\{B_{i}\left(\theta_{f, w}\right) \frac{d \bar{\omega}_{f}\left(\bar{T}_{f, w}\right)}{d \theta_{f, w}(r)}+\frac{d i_{a}\left(\theta_{f, w}\right)}{d \theta_{f, w}(r)}\left[\bar{\omega}_{f}\left(\bar{T}_{f, w}\right)-\bar{\omega}_{h}\right]\right\} \\
& =2\left[\mathrm{Bi}_{\mathrm{a}}\left(\theta_{\mathrm{f}, \mathrm{w}}\right)+\frac{\mathrm{dBi}_{\mathrm{a}}\left(\theta_{\mathrm{f}, \mathrm{w}}\right)}{\mathrm{d} \theta_{\mathrm{f}, \mathrm{w}}(\mathrm{r})} \theta_{\mathrm{f}, \mathrm{w}}(\mathrm{r})\right] \lambda_{\mathrm{f}, \mathrm{w}}(\mathrm{r}) \\
& +2 \bar{h}_{\mathrm{fg}} \frac{\left(\mathrm{C}_{\mathrm{p}, \mathrm{a}} \mathrm{L}_{\mathrm{e}}{ }^{2 / 3}\right)^{-1}}{\left(\overline{\mathrm{T}}_{\mathrm{c}}-\overline{\mathrm{T}}_{\mathrm{h}}\right)}\left[\mathrm{Bi}_{\mathrm{a}}\left(\theta_{\mathrm{f}, \mathrm{w}}\right) \frac{\mathrm{d} \bar{\omega}_{\mathrm{f}}\left(\overline{\mathrm{T}}_{\mathrm{f}, \mathrm{w}}\right)}{\mathrm{d} \theta_{\mathrm{f}, \mathrm{w}}(\mathrm{r})}+\frac{\mathrm{dBi} \mathrm{i}_{\mathrm{a}}\left(\theta_{\mathrm{f}, \mathrm{w}}\right)}{\mathrm{d} \theta_{\mathrm{f}, \mathrm{w}}(\mathrm{r})}\left(\bar{\omega}_{\mathrm{f}}\left(\overline{\mathrm{T}}_{\mathrm{f}, \mathrm{w}}\right)-\bar{\omega}_{\mathrm{h}}\right)\right] \lambda_{\mathrm{f}, \mathrm{w}}(\mathrm{r}) ; \text { in } \mathrm{r}_{\mathrm{o}} \leq \mathrm{r} \leq \mathrm{r}_{\mathrm{wd}} \\
& \mathrm{k}_{\mathrm{f}}\left(\theta_{\mathrm{f}, \mathrm{d}}\right) \frac{1}{\mathrm{r}} \frac{\mathrm{d}}{\mathrm{dr}}\left[\mathrm{r} \delta(\mathrm{r}) \frac{\mathrm{d} \lambda_{\mathrm{f}, \mathrm{d}}(\mathrm{r})}{\mathrm{dr}}\right]+8 \pi \mathrm{r}(\mathrm{q}-\Phi \mathrm{Q})\left[\mathrm{Bi}_{\mathrm{a}}\left(\theta_{\mathrm{f}, \mathrm{d}}\right)+\frac{\mathrm{dBi} \mathrm{i}_{\mathrm{a}}\left(\theta_{\mathrm{f}, \mathrm{d}}\right)}{\mathrm{d} \theta_{\mathrm{f}, \mathrm{d}}(\mathrm{r})} \theta_{\mathrm{f}, \mathrm{d}}(\mathrm{r})\right] \\
& =2\left[\operatorname{Bi}_{\mathrm{a}}\left(\theta_{\mathrm{f}, \mathrm{d}}\right)+\frac{\mathrm{dBi}_{\mathrm{a}}\left(\theta_{\mathrm{f}, \mathrm{d}}\right)}{\mathrm{d} \theta_{\mathrm{f}, \mathrm{d}}(\mathrm{r})} \theta_{\mathrm{f}, \mathrm{d}}(\mathrm{r})\right] \lambda_{\mathrm{f}, \mathrm{d}}(\mathrm{r}) \\
& \text {; in } \mathrm{r}_{\mathrm{wd}} \leq \mathrm{r} \leq 1 \\
& k_{w}\left(\theta_{w}\right) \frac{d \lambda_{w}}{d r}=\left[\frac{1}{k_{w}\left(\theta_{w}\right)} \frac{d k_{w}\left(\theta_{w}\right)}{d \theta_{w}} B_{i}\left(\theta_{w}\right)\left(1-\theta_{W}\right)-\frac{d k_{w}\left(\theta_{w}\right)}{d \theta_{w}} \frac{d \theta_{w}}{d r}\right. \\
& \left.-\mathrm{Bi}_{\mathrm{i}}\left(\theta_{\mathrm{w}}\right)-\frac{\mathrm{dB} i_{\mathrm{i}}\left(\theta_{\mathrm{w}}\right)}{\mathrm{d} \theta_{\mathrm{w}}}\left(1-\theta_{\mathrm{w}}\right)\right] \lambda_{\mathrm{w}} ; \text { at } \mathrm{r}=\mathrm{r}_{\mathrm{b}} \\
& \frac{1}{2 \mathrm{P}} \lambda_{\mathrm{w}}=\lambda_{\mathrm{f}, \mathrm{w}} ; \text { at } \mathrm{r}=\mathrm{r}_{\mathrm{o}} \\
& -\mathrm{k}_{\mathrm{w}}\left(\theta_{\mathrm{w}}\right) \mathrm{P} \frac{\mathrm{d} \lambda_{\mathrm{w}}}{\mathrm{dr}}=\left(\mathrm{P}-\frac{1}{2} \delta\right)\left[\frac{\mathrm{dBi} i_{\mathrm{a}}\left(\theta_{\mathrm{f}, \mathrm{w}}\right)}{\mathrm{d} \theta_{\mathrm{f}, \mathrm{w}}} \theta_{\mathrm{f}, \mathrm{w}}+\mathrm{Bi}_{\mathrm{a}}\left(\theta_{\mathrm{f}, \mathrm{w}}\right)\right] \lambda_{\mathrm{w}} \\
& +\left(P-\frac{1}{2} \delta\right) \bar{h}_{f g} \frac{\left(C_{p, a} L_{e}{ }^{2 / 3}\right)^{-1}}{\left(\bar{T}_{c}-\bar{T}_{h}\right)}\left[\left(\bar{\omega}_{f}\left(\bar{T}_{f, w}\right)-\bar{\omega}_{h}\right) \frac{d i_{a}\left(\theta_{f, w}\right)}{d \theta_{f, w}}+B i_{a}\left(\theta_{f, w}\right) \frac{d \bar{\omega}_{f}\left(\bar{T}_{f, w}\right)}{d \theta_{f, w}}\right] \lambda_{w} \\
& -\mathrm{Pk}_{\mathrm{f}}\left(\theta_{\mathrm{f}, \mathrm{w}}\right) \delta(\mathrm{r}) \frac{\mathrm{d} \lambda_{\mathrm{f}, \mathrm{w}}}{\mathrm{dr}}+4 \pi(-\Phi) \mathrm{P}(\mathrm{q}-\mathrm{Q})\left\{\mathrm{Bi}_{\mathrm{a}}\left[\theta_{\mathrm{f}}\left(\mathrm{r}_{o}\right)\right]\left[1+\frac{\overline{\mathrm{h}}_{\mathrm{fg}}\left(\mathrm{C}_{\mathrm{p}, \mathrm{a}} \mathrm{L}_{\mathrm{e}}{ }^{2 / 3}\right)^{-1}}{\left(\overline{\mathrm{T}}_{\mathrm{c}}-\overline{\mathrm{T}}_{\mathrm{h}}\right)} \frac{\mathrm{d} \bar{\omega}_{\mathrm{f}}\left(\overline{\mathrm{T}}_{\mathrm{o}}\right)}{\mathrm{d} \theta_{f}\left(\mathrm{r}_{o}\right)}\right]\right. \\
& \left.+\frac{\mathrm{dBi}_{\mathrm{a}}\left[\theta_{\mathrm{f}}\left(\mathrm{r}_{o}\right)\right]}{\mathrm{d} \theta_{\mathrm{f}}\left(\mathrm{r}_{o}\right)} \theta_{f}\left(\mathrm{r}_{o}\right)+\frac{\overline{\mathrm{h}}_{\mathrm{fg}}\left(\mathrm{C}_{\mathrm{p}, \mathrm{a}} \mathrm{L}_{\mathrm{e}}{ }^{2 / 3}\right)^{-1}}{\left(\overline{\mathrm{T}}_{\mathrm{c}}-\overline{\mathrm{T}}_{\mathrm{h}}\right)} \frac{\mathrm{dBi}_{\mathrm{a}}\left[\theta_{\mathrm{f}}\left(\mathrm{r}_{o}\right)\right]}{\mathrm{d} \theta_{\mathrm{f}}\left(\mathrm{r}_{o}\right)}\left[\bar{\omega}_{\mathrm{f}}\left(\overline{\mathrm{T}}_{\mathrm{o}}\right)-\bar{\omega}_{\mathrm{h}}\right]\right\} \frac{1}{\mathrm{r}}\left(\mathrm{r}_{\mathrm{wd}}{ }^{2}-\mathrm{r}_{\mathrm{o}}{ }^{2}\right) \\
& +4 \pi(-\Phi) \mathrm{P}(\mathrm{q}-\mathrm{Q})\left\{\mathrm{Bi}_{\mathrm{a}}\left[\theta_{\mathrm{f}}\left(\mathrm{r}_{o}\right)\right]+\frac{\mathrm{dBi}_{\mathrm{a}}\left[\theta_{\mathrm{f}}\left(\mathrm{r}_{o}\right)\right]}{\mathrm{d} \theta_{\mathrm{f}}\left(\mathrm{r}_{o}\right)} \theta_{f}\left(\mathrm{r}_{o}\right)\right\} \frac{1}{\mathrm{r}}\left(1-\mathrm{r}_{\mathrm{wd}}{ }^{2}\right) \text { at } \mathrm{r}=\mathrm{r}_{o}
\end{aligned}
$$




$$
\begin{aligned}
& \lambda_{\mathrm{f}, \mathrm{w}}=\lambda_{\mathrm{f}, \mathrm{d}} ; \text { at } \mathrm{r}=\mathrm{r}_{\mathrm{wd}} \\
& \frac{\mathrm{d} \lambda_{\mathrm{f}, \mathrm{w}}}{\mathrm{dr}}=\frac{\mathrm{d} \lambda_{\mathrm{f}, \mathrm{d}}}{\mathrm{dr}} ; \text { at } \mathrm{r}=\mathrm{r}_{\mathrm{wd}} \\
& k_{f}\left(\theta_{f, d}\right) \frac{d \lambda_{f, d}(r)}{d r}=\left\{\frac{d k_{f}\left(\theta_{f, d}\right)}{d r}+\frac{d k_{f}\left(\theta_{f, d}\right)}{d \theta_{f, d}} \frac{d \theta_{f, d}}{d r}+B i_{a}\left(\theta_{f, d}\right)+\frac{d B i_{a}\left(\theta_{f, d}\right)}{d \theta_{f, d}} \theta_{f, d}\right\} \lambda_{f, d}(r) ; \text { at } r=1
\end{aligned}
$$

Finally, the following integral terms are left:

$$
\begin{aligned}
& \Delta \mathrm{J}(\delta)=\int_{\mathrm{r}=\mathrm{r}_{\mathrm{o}}}^{1}\{4 \pi \alpha(\mathrm{v}-\mathrm{V}) \\
& \quad-\left[\mathrm{k}_{\mathrm{f}}\left(\theta_{\mathrm{f}, \mathrm{w}}\right) \frac{\mathrm{d} \lambda_{\mathrm{f}, \mathrm{w}}(\mathrm{r})}{\mathrm{dr}} \frac{\mathrm{d} \theta_{\mathrm{f}, \mathrm{w}}(\mathrm{r})}{\mathrm{dr}}\right]\left[\mathrm{u}\left(\mathrm{r}-\mathrm{r}_{\mathrm{o}}\right)-\mathrm{u}\left(\mathrm{r}-\mathrm{r}_{\mathrm{wd}}\right)\right] \\
& \quad-\left[\mathrm{k}_{\mathrm{f}}\left(\theta_{\mathrm{f}, \mathrm{d}}\right) \frac{\mathrm{d} \lambda_{\mathrm{f}, \mathrm{d}}(\mathrm{r})}{\mathrm{dr}} \frac{\mathrm{d} \theta_{\mathrm{f,d}}(\mathrm{r})}{\mathrm{dr}}\right]\left[\mathrm{u}\left(\mathrm{r}-\mathrm{r}_{\mathrm{wd}}\right)-\mathrm{u}(\mathrm{r}-1)\right] \\
& \quad+\lambda_{\mathrm{f}, \mathrm{w}}(\mathrm{r}) B_{\mathrm{a}}\left(\theta_{\mathrm{f}, \mathrm{w}}\right)\left\{\theta_{\mathrm{f}, \mathrm{w}}+\overline{\mathrm{h}}_{\mathrm{fg}} \frac{\left(\mathrm{C}_{\mathrm{p}, \mathrm{a}} \mathrm{L}_{\mathrm{e}} \mathrm{e}^{2 / 3}\right)^{-1}}{\left(\overline{\mathrm{T}}_{\mathrm{c}}-\overline{\mathrm{T}}_{\mathrm{h}}\right)}\left[\overline{\mathrm{\omega}}_{\mathrm{f}}\left(\overline{\mathrm{T}}_{\mathrm{f}, \mathrm{w}}\right)-\overline{\mathrm{\omega}}_{\mathrm{h}}\right]\right\} \Gamma\left(\mathrm{r}-\mathrm{r}_{\mathrm{o}}\right) \\
& \left.\quad+\lambda_{\mathrm{f}, \mathrm{d}}(\mathrm{r}) \mathrm{k}_{\mathrm{f}}\left(\theta_{\mathrm{f}, \mathrm{d}}\right) \frac{\mathrm{d} \theta_{\mathrm{f}, \mathrm{d}}(\mathrm{r})}{\mathrm{dr}} \Gamma(\mathrm{r}-1)\right\} \mathrm{r} \Delta \delta(\mathrm{r}) \mathrm{dr}
\end{aligned}
$$

Here $\mathrm{u}($.$) and \Gamma($.$) denote the unit step and Dirac delta functions, respectively.$ The perturbation of cost function can be presented as (Alifanov [17]):

$$
\Delta \mathrm{J}(\delta)=\int_{\mathrm{r}=\mathrm{r}_{\mathrm{o}}}^{1} \mathrm{~J}^{\prime}(\delta) \mathrm{r} \Delta \delta(\mathrm{r}) \mathrm{dr}
$$

The expression for the gradient $J^{\prime}(\delta)$ is obtained by comparing Equations (14a) and $(14 \mathrm{~b})$ and is given below:

$$
\begin{aligned}
& \mathrm{J}^{\prime}(\mathrm{r})=4 \pi \alpha(\mathrm{v}-\mathrm{V}) \\
& \quad-\left[\mathrm{k}_{\mathrm{f}}\left(\theta_{\mathrm{f}, \mathrm{w}}\right) \frac{\mathrm{d} \lambda_{\mathrm{f}, \mathrm{w}}(\mathrm{r})}{\mathrm{dr}} \frac{\mathrm{d} \theta_{\mathrm{f}, \mathrm{w}}(\mathrm{r})}{\mathrm{dr}}\right]\left[\mathrm{u}\left(\mathrm{r}-\mathrm{r}_{\mathrm{o}}\right)-\mathrm{u}\left(\mathrm{r}-\mathrm{r}_{\mathrm{wd}}\right)\right] \\
& \quad-\left[\mathrm{k}_{\mathrm{f}}\left(\theta_{\mathrm{f}, \mathrm{d}}\right) \frac{\mathrm{d} \lambda_{\mathrm{f}, \mathrm{d}}(\mathrm{r})}{\mathrm{dr}} \frac{\mathrm{d} \theta_{\mathrm{f}, \mathrm{d}}(\mathrm{r})}{\mathrm{dr}}\right]\left[\mathrm{u}\left(\mathrm{r}-\mathrm{r}_{\mathrm{wd}}\right)-\mathrm{u}(\mathrm{r}-1)\right] \\
& \quad+\lambda_{\mathrm{f}, \mathrm{w}}(\mathrm{r}) \operatorname{Bi}_{\mathrm{a}}\left(\theta_{\mathrm{f}, \mathrm{w}}\right)\left\{\theta_{\mathrm{f}, \mathrm{w}}+\overline{\mathrm{h}}_{\mathrm{fg}} \frac{\left(\mathrm{C}_{\mathrm{p}, \mathrm{a}} \mathrm{L}_{\mathrm{e}}{ }^{2 / 3}\right)^{-1}}{\left(\bar{T}_{\mathrm{c}}-\bar{T}_{\mathrm{h}}\right)}\left[\bar{\omega}_{\mathrm{f}}\left(\bar{T}_{\mathrm{f}, \mathrm{w}}\right)-\bar{\omega}_{\mathrm{h}}\right]\right\} \Gamma\left(\mathrm{r}-\mathrm{r}_{\mathrm{o}}\right) \\
& \quad+\lambda_{\mathrm{f}, \mathrm{d}}(\mathrm{r}) \mathrm{k}_{\mathrm{f}}\left(\theta_{\mathrm{f}, \mathrm{d}}\right) \frac{\mathrm{d} \theta_{\mathrm{f}, \mathrm{d}}(\mathrm{r})}{\mathrm{dr}} \Gamma(\mathrm{r}-1)
\end{aligned}
$$

\section{Results and Discussion}

The aim of this study was to estimate the optimal shapes for partially wet annular fins under the condition that $\mathrm{k}_{\mathrm{w}}, \mathrm{k}_{\mathrm{f}}, \mathrm{Bi}_{\mathrm{i}}$, and $\mathrm{Bi}_{\mathrm{a}}$ of the fin are functions of temperature. In the present fin design problem, an iterative algorithm of CGM is utilized, and the minimization process is based on $\Phi$ and V. In the numerical calculations, the space increment is equal to $\Delta \mathrm{r}=0.002$, and $\mathrm{r}_{\mathrm{b}}, \mathrm{r}_{\mathrm{o}}$, and $\mathrm{r}_{\mathrm{t}}$ are given as $0.2,0.4$ and 1.0, respectively. In addition, a relative humidity of $\phi=0.8,0.85$, and 0.9 and $\mathrm{P}=0.13$ are utilized in this study.

The numerical solutions for the rectangular, triangular, concave, convex and hyperbolic types non-linear partially wet annular fin efficiencies are computed and summarized in Table 1 for various $\phi, \mathrm{k}_{\mathrm{w}}, \mathrm{k}_{\mathrm{f}}$, and $B \mathrm{i}_{\mathrm{i}}$, i.e., $\phi=0.8,0.85,0.9 ; \mathrm{k}_{\mathrm{w}}=1$ and $1+0.1 \theta_{\mathrm{w}}$; $\mathrm{k}_{\mathrm{f}}=1$ and $1+0.1 \theta_{\mathrm{f}} ; \mathrm{Bi}_{\mathrm{i}}=4$ and $4+0.1 \theta_{\mathrm{w}} ;$ and $\mathrm{Bi}_{\mathrm{a}}=0.3$ and $0.3+0.1 \theta_{\mathrm{f}}$. It is observed from Table 1 that the computed fin efficiency $\eta$ increases as the relative humidity $\phi$ increases, the thermal conductivities $\mathrm{k}_{\mathrm{w}}$ and $\mathrm{k}_{\mathrm{f}}$ increase, $\mathrm{Bi}_{\mathrm{i}}$ increases, and $\mathrm{Bi}_{\mathrm{a}}$ decreases. In addition, the interfacial radii $r_{w d}$ between the wet-dry fin domains for the previous examples are also calculated and listed in Table 2. 
Table 1. The numerical solutions of $\eta$ for different types of fins with various $\phi, \mathrm{k}_{\mathrm{w}}, \mathrm{k}_{\mathrm{f}}, \mathrm{Bi}_{\mathrm{i}}$, and $\mathrm{Bi}_{\mathrm{a}}$.

\begin{tabular}{|c|c|c|c|c|c|c|c|c|c|c|c|c|c|c|c|}
\hline & & & & & & & & & & & 1 & & & & $\begin{array}{l}\text { Number } \\
\text { of Iterative }\end{array}$ \\
\hline$\Phi$ & $\mathbf{V}$ & $\alpha$ & $\phi$ & kw & $\mathbf{k f}$ & Bii & Bia & $\mathbf{V}$ & Rectangular & Triangular & Concave & Convex & Hyperbolic & Optimal & $\mathbf{N}$ \\
\hline \multirow{24}{*}{0.3} & \multirow{24}{*}{0.08} & \multirow{24}{*}{625} & \multirow{8}{*}{0.8} & \multirow{4}{*}{1} & \multirow{4}{*}{1} & \multirow{2}{*}{4} & 0.3 & & 0.1472 & 0.2086 & 0.2450 & 0.1726 & 0.1815 & 0.2624 & 425 \\
\hline & & & & & & & $0.3+0.1 \theta_{\mathrm{f}}$ & & 0.1336 & 0.1924 & 0.2272 & 0.1576 & 0.1661 & 0.2443 & 382 \\
\hline & & & & & & \multirow{2}{*}{$4+0.1 \theta_{\mathrm{w}}$} & 0.3 & & 0.1473 & 0.2088 & 0.2452 & 0.1727 & 0.1817 & 0.2625 & 556 \\
\hline & & & & & & & $0.3+0.1 \theta_{\mathrm{f}}$ & & 0.1338 & 0.1927 & 0.2275 & 0.1578 & 0.1663 & 0.2445 & 448 \\
\hline & & & & \multirow{4}{*}{$1+0.1 \theta_{\mathrm{w}}$} & \multirow{4}{*}{$1+0.1 \theta_{\mathrm{f}}$} & \multirow{2}{*}{4} & 0.3 & & 0.1497 & 0.2117 & 0.2483 & 0.1753 & 0.1843 & 0.2659 & 326 \\
\hline & & & & & & & $0.3+0.1 \theta_{\mathrm{f}}$ & & 0.1355 & 0.1945 & 0.2302 & 0.1601 & 0.1683 & 0.2466 & 201 \\
\hline & & & & & & \multirow{2}{*}{$4+0.1 \theta_{\mathrm{w}}$} & 0.3 & & 0.1498 & 0.2119 & 0.2485 & 0.1755 & 0.1845 & 0.2662 & 710 \\
\hline & & & & & & & $0.3+0.1 \theta_{\mathrm{f}}$ & & 0.1357 & 0.1947 & 0.2305 & 0.1604 & 0.1686 & 0.2468 & 365 \\
\hline & & & \multirow{8}{*}{0.85} & \multirow{4}{*}{1} & \multirow{4}{*}{1} & \multirow{2}{*}{4} & 0.3 & 0.08 & 0.1478 & 0.2117 & 0.2488 & 0.1774 & 0.1830 & 0.2682 & 476 \\
\hline & & & & & & & $0.3+0.1 \theta_{\mathrm{f}}$ & & 0.1342 & 0.1955 & 0.2305 & 0.1624 & 0.1675 & 0.2501 & 655 \\
\hline & & & & & & \multirow{2}{*}{$4+0.1 \theta_{\mathrm{w}}$} & 0.3 & & 0.1479 & 0.2119 & 0.2490 & 0.1775 & 0.1832 & 0.2684 & 399 \\
\hline & & & & & & & $0.3+0.1 \theta_{\mathrm{f}}$ & & 0.1344 & 0.1958 & 0.2308 & 0.1627 & 0.1678 & 0.2509 & 395 \\
\hline & & & & \multirow{4}{*}{$1+0.1 \theta_{\mathrm{w}}$} & \multirow{4}{*}{$1+0.1 \theta_{\mathrm{f}}$} & \multirow{2}{*}{4} & 0.3 & & 0.1503 & 0.2148 & 0.2521 & 0.1801 & 0.1858 & 0.2721 & 549 \\
\hline & & & & & & & $0.3+0.1 \theta_{\mathrm{f}}$ & & 0.1363 & 0.1974 & 0.2334 & 0.1651 & 0.1699 & 0.2528 & 307 \\
\hline & & & & & & \multirow{2}{*}{$4+0.1 \theta_{\mathrm{w}}$} & 0.3 & & 0.1504 & 0.2150 & 0.2523 & 0.1803 & 0.1860 & 0.2725 & 811 \\
\hline & & & & & & & $0.3+0.1 \theta_{\mathrm{f}}$ & & 0.1365 & 0.1977 & 0.2337 & 0.1654 & 0.1702 & 0.2537 & 388 \\
\hline & & & \multirow{8}{*}{0.9} & \multirow{4}{*}{1} & \multirow{4}{*}{1} & \multirow{2}{*}{4} & 0.3 & & 0.1482 & 0.2139 & 0.2508 & 0.1792 & 0.1838 & 0.2704 & 664 \\
\hline & & & & & & & $0.3+0.1 \theta_{\mathrm{f}}$ & & 0.1346 & 0.1967 & 0.2325 & 0.1642 & 0.1684 & 0.2514 & 582 \\
\hline & & & & & & \multirow{2}{*}{$4+0.1 \theta_{\mathrm{w}}$} & 0.3 & & 0.1483 & 0.2142 & 0.2511 & 0.1794 & 0.1840 & 0.2710 & 486 \\
\hline & & & & & & & $0.3+0.1 \theta_{\mathrm{f}}$ & & 0.1347 & 0.1970 & 0.2328 & 0.1644 & 0.1687 & 0.2521 & 421 \\
\hline & & & & \multirow{4}{*}{$1+0.1 \theta_{\mathrm{w}}$} & \multirow{4}{*}{$1+0.1 \theta_{\mathrm{f}}$} & 4 & 0.3 & & 0.1507 & 0.2170 & 0.2541 & 0.1819 & 0.1866 & 0.2740 & 628 \\
\hline & & & & & & & $0.3+0.1 \theta_{\mathrm{f}}$ & & 0.1367 & 0.1994 & 0.2354 & 0.1666 & 0.1707 & 0.2550 & 766 \\
\hline & & & & & & $+01 \theta_{u}$ & 0.3 & & 0.1508 & 0.2172 & 0.2543 & 0.1821 & 0.1867 & 0.2742 & 729 \\
\hline & & & & & & $2-8.00 \mathrm{w}$ & $0.3+0.1 \theta_{\mathrm{f}}$ & & 0.1369 & 0.1996 & 0.2356 & 0.1669 & 0.1711 & 0.2557 & 606 \\
\hline
\end{tabular}


Table 2. The numerical solutions of wet-dry interface $\mathrm{r}_{\mathrm{wd}}$ for different types of fins. Various $\phi, \mathrm{k}_{\mathrm{w}}, \mathrm{k}_{\mathrm{f}}, \mathrm{Bi}_{\mathrm{i}}$, and $\mathrm{Bi}_{\mathrm{a}}$.

\begin{tabular}{|c|c|c|c|c|c|c|c|c|c|c|c|c|c|c|}
\hline \multirow[b]{2}{*}{$\Phi$} & \multirow[b]{2}{*}{$\mathbf{V}$} & \multirow[b]{2}{*}{$\alpha$} & \multirow[b]{2}{*}{$\phi$} & \multirow[b]{2}{*}{ kf } & \multirow[b]{2}{*}{ kw } & \multirow[b]{2}{*}{ Bii } & \multirow[b]{2}{*}{ Bia } & \multirow[b]{2}{*}{$\mathbf{V}$} & \multicolumn{6}{|c|}{ Rwd } \\
\hline & & & & & & & & & Rectangular & Triangular & Concave & Convex & Hyperbolic & Optimal \\
\hline \multirow{24}{*}{0.3} & \multirow{24}{*}{0.08} & \multirow{24}{*}{625} & \multirow{8}{*}{0.8} & \multirow{4}{*}{1} & \multirow{4}{*}{1} & \multirow{2}{*}{4} & 0.3 & \multirow{24}{*}{0.08} & 0.521 & 0.573 & 0.606 & 0.547 & 0.552 & 0.637 \\
\hline & & & & & & & $0.3+0.1 \theta_{\mathrm{f}}$ & & 0.511 & 0.555 & 0.591 & 0.532 & 0.540 & 0.620 \\
\hline & & & & & & \multirow{2}{*}{$4+0.1 \theta_{\mathrm{w}}$} & 0.3 & & 0.521 & 0.574 & 0.607 & 0.548 & 0.553 & 0.638 \\
\hline & & & & & & & $0.3+0.1 \theta_{\mathrm{f}}$ & & 0.512 & 0.556 & 0.592 & 0.533 & 0.541 & 0.621 \\
\hline & & & & \multirow{4}{*}{$1+0.1 \theta_{\mathrm{f}}$} & \multirow{4}{*}{$1+0.1 \theta_{\mathrm{w}}$} & \multirow{2}{*}{4} & 0.3 & & 0.524 & 0.576 & 0.610 & 0.551 & 0.556 & 0.641 \\
\hline & & & & & & & $0.3+0.1 \theta_{\mathrm{f}}$ & & 0.514 & 0.558 & 0.595 & 0.535 & 0.543 & 0.625 \\
\hline & & & & & & \multirow{2}{*}{$4+0.1 \theta_{\mathrm{w}}$} & 0.3 & & 0.524 & 0.578 & 0.611 & 0.552 & 0.557 & 0.642 \\
\hline & & & & & & & $0.3+0.1 \theta_{\mathrm{f}}$ & & 0.515 & 0.559 & 0.596 & 0.540 & 0.544 & 0.627 \\
\hline & & & \multirow{8}{*}{0.85} & \multirow{4}{*}{1} & \multirow{4}{*}{1} & \multirow{2}{*}{4} & 0.3 & & 0.561 & 0.616 & 0.655 & 0.592 & 0.589 & 0.688 \\
\hline & & & & & & & $0.3+0.1 \theta_{\mathrm{f}}$ & & 0.546 & 0.610 & 0.643 & 0.580 & 0.584 & 0.672 \\
\hline & & & & & & \multirow{2}{*}{$4+0.1 \theta_{\mathrm{w}}$} & 0.3 & & 0.562 & 0.617 & 0.656 & 0.593 & 0.590 & 0.689 \\
\hline & & & & & & & $0.3+0.1 \theta_{\mathrm{f}}$ & & 0.546 & 0.611 & 0.644 & 0.581 & 0.585 & 0.673 \\
\hline & & & & & & 4 & 0.3 & & 0.564 & 0.619 & 0.660 & 0.596 & 0.593 & 0.692 \\
\hline & & & & $1+018$ & $1+019$ & & $0.3+0.1 \theta_{\mathrm{f}}$ & & 0.549 & 0.614 & 0.646 & 0.584 & 0.587 & 0.676 \\
\hline & & & & $1+0.10_{\mathrm{f}}$ & $1+0.10_{\mathrm{w}}$ & $4+0.1 \theta_{W}$ & 0.3 & & 0.565 & 0.520 & 0.661 & 0.597 & 0.593 & 0.693 \\
\hline & & & & & & $2.0 .00 \mathrm{~W}$ & $0.3+0.1 \theta_{\mathrm{f}}$ & & 0.549 & 0.615 & 0.648 & 0.585 & 0.588 & 0.678 \\
\hline & & & \multirow{8}{*}{0.9} & & & 4 & 0.3 & & 0.612 & 0.677 & 0.714 & 0.651 & 0.642 & 0.726 \\
\hline & & & & & & & $0.3+0.1 \theta_{\mathrm{f}}$ & & 0.601 & 0.675 & 0.703 & 0.639 & 0.632 & 0.709 \\
\hline & & & & 1 & 1 & $4+0.1 \theta_{W}$ & 0.3 & & 0.613 & 0.678 & 0.715 & 0.652 & 0.643 & 0.727 \\
\hline & & & & & & & $0.3+0.1 \theta_{\mathrm{f}}$ & & 0.601 & 0.676 & 0.704 & 0.640 & 0.633 & 0.711 \\
\hline & & & & & & 4 & 0.3 & & 0.615 & 0.681 & 0.717 & 0.655 & 0.646 & 0.730 \\
\hline & & & & $1+010$ & 1 & & $0.3+0.1 \theta_{\mathrm{f}}$ & & 0.604 & 0.679 & 0.706 & 0.643 & 0.635 & 0.714 \\
\hline & & & & $1+0.10_{\mathrm{f}}$ & $1+0.10_{\mathrm{W}}$ & $4+01 \theta_{w}$ & 0.3 & & 0.616 & 0.682 & 0.718 & 0.656 & 0.647 & 0.731 \\
\hline & & & & & & $2.0 .00 \mathrm{~W}$ & $0.3+0.1 \theta_{\mathrm{f}}$ & & 0.605 & 0.680 & 0.707 & 0.644 & 0.636 & 0.716 \\
\hline
\end{tabular}


Now, let us explore the reasons behind those observations. Table 1 indicates that when $\phi$ increases, $\eta$ also increases. The reason is that a higher value of $\phi$ indicates a lower fin surface temperature, which is closer to the fin base temperature, therefore, the fin will have a higher $\eta$. This result is similar to that reported by Huang and Chung [20]. Similarly, higher $\mathrm{k}_{\mathrm{w}}, \mathrm{k}_{\mathrm{f}}, \mathrm{Bi}_{\mathrm{i}}$, and lower $\mathrm{Bi}_{\mathrm{a}}$ will result in a lower fin surface temperature, therefore yielding higher $\eta$.

It can be seen from Table 2 that the value of $r_{w d}$ increases as $\phi$ increases, $k_{w}$ and $k_{f}$ increase, $\mathrm{Bi}_{\mathrm{i}}$ increases, and $\mathrm{Bi}_{\mathrm{a}}$ decreases. It is because as $\mathrm{r}_{\mathrm{wd}}$ increases, the fin surface temperature approaches the fin base temperature; as a result, $\eta$ becomes higher. Tables 1 and 2 show that the fin profiles with rectangular and concave shapes have the shortest and longest $r_{w d}$, respectively, which also implies that they have the lowest and highest fin efficiencies, respectively, among these five common annular fin shapes.

The rectangular annular fin shape is considered as the initial design of this work. Figure 2a plots a quarter of a rectangular fin; to have better visibility, Figure $2 b$ illustrates the whole rectangular shape of a annular fin with the following system parameters: $\mathrm{V}=0.08$, $\phi=0.85, \mathrm{k}_{\mathrm{w}}=1+0.1 \theta_{\mathrm{w}}, \mathrm{k}_{\mathrm{f}}=1+0.1 \theta_{\mathrm{f}}, \mathrm{Bi}_{\mathrm{i}}=4+0.1 \theta_{\mathrm{w}}$, and $\mathrm{Bi}_{\mathrm{a}}=0.3+0.1 \theta_{\mathrm{f}}$. In Figure $2 \mathrm{a}$, the dashed line represents the interface between the dry and wet regions of fin.

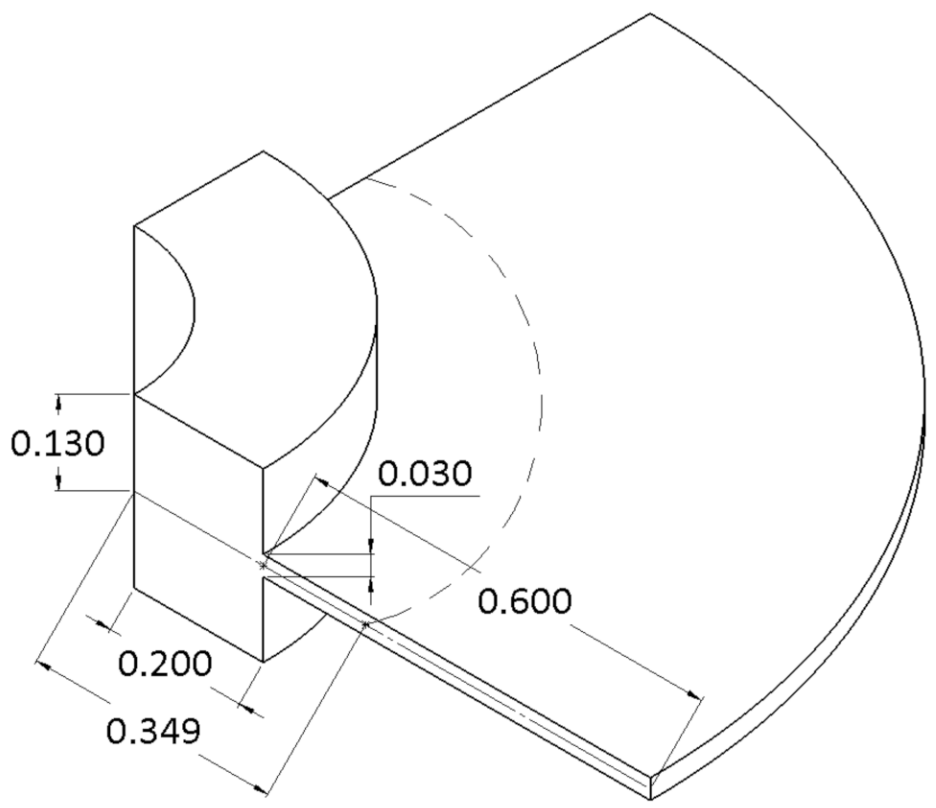

(a)

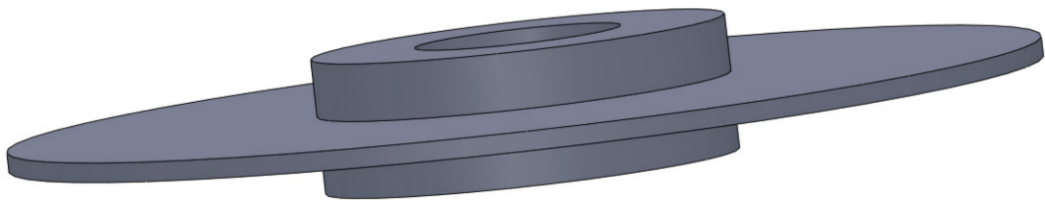

(b)

Figure 2. (a) The dimensions of a quarter of a single rectangular shape annular fin. (b) A complete rectangular shape annular fin of Figure 2a.

To investigate the influence of the non-linear physical properties on the temperature variation of the partially wet fins, the following numerical solutions of the direct problem with various physical properties are conducted, and the computational solutions for the dimensionless temperatures are plotted in Figures 3 and 4. 


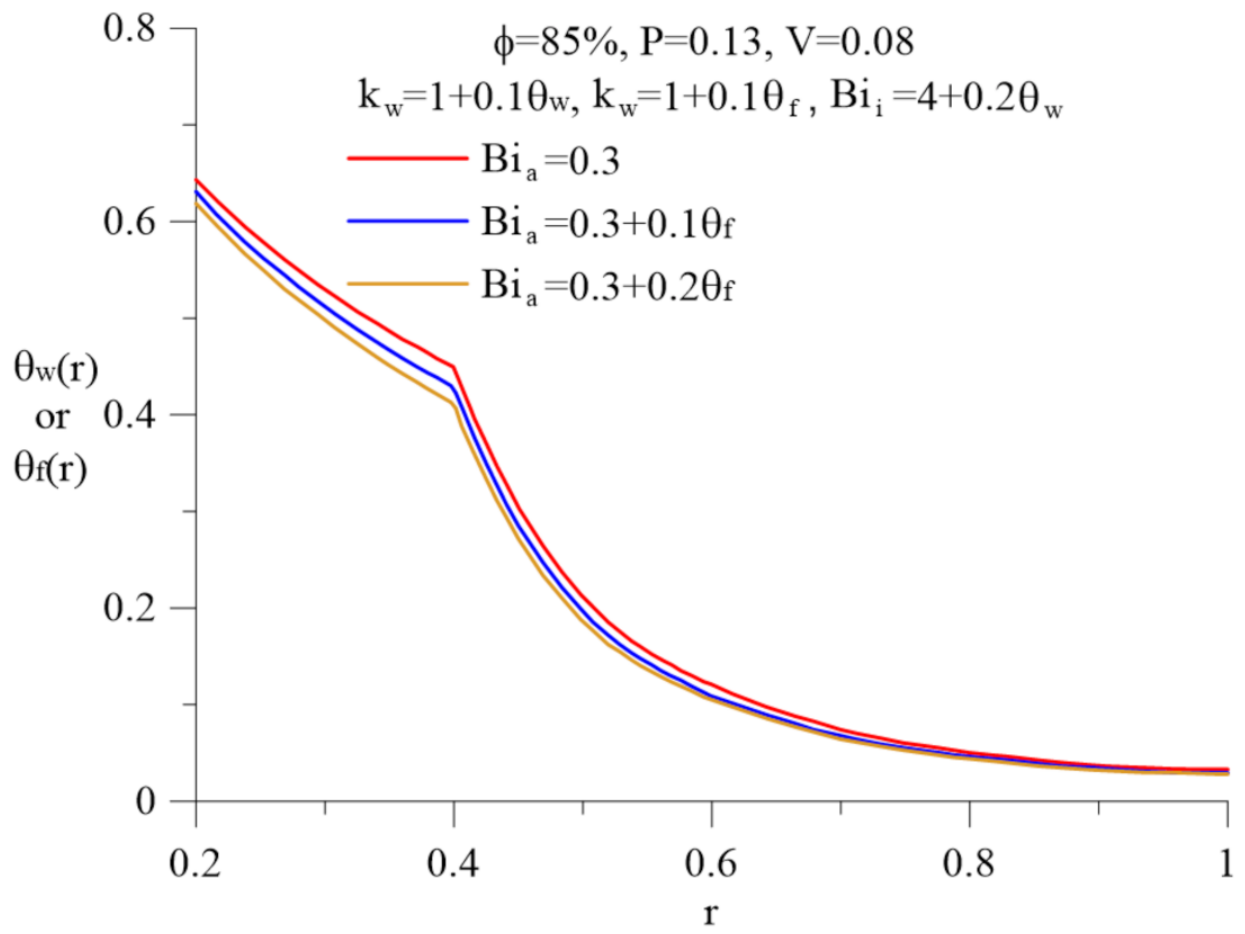

(a)

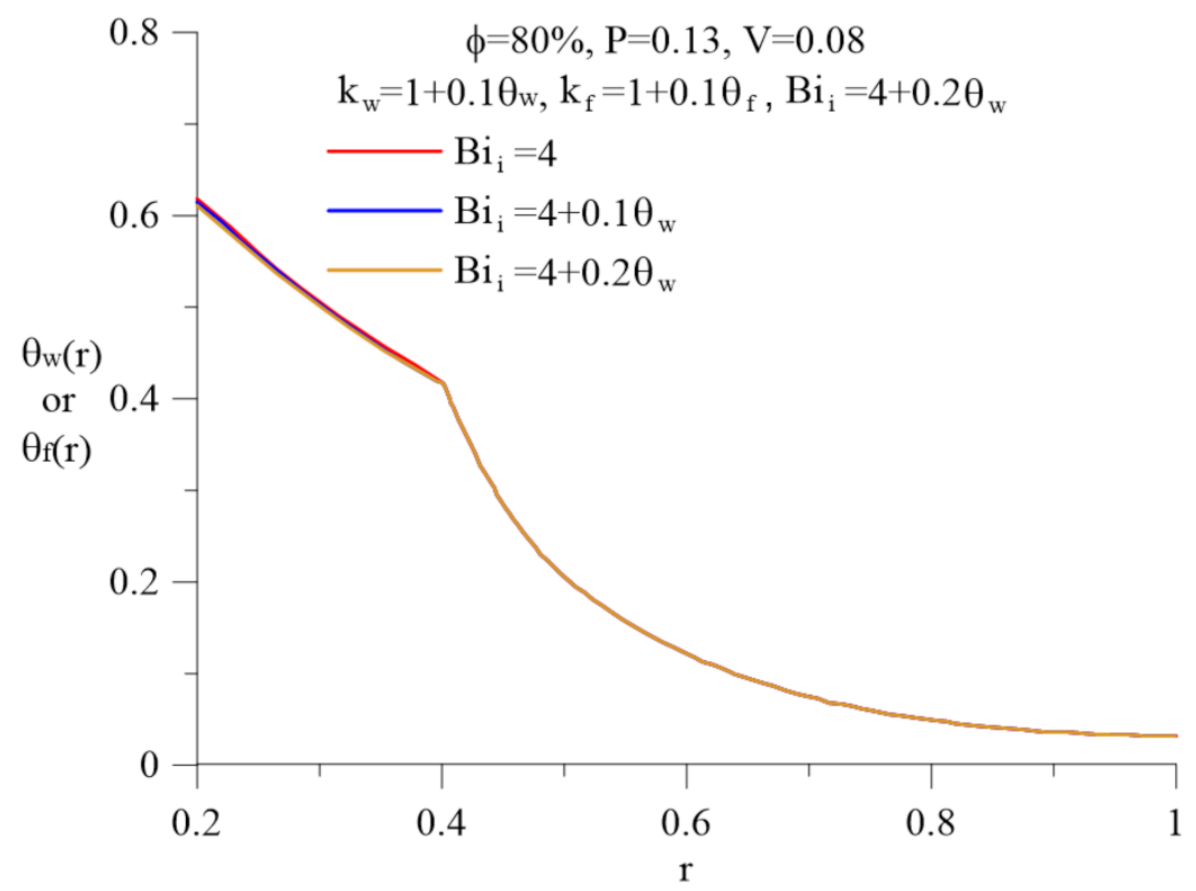

(b)

Figure 3. (a) The temperature variations of a rectangular fin with various $\mathrm{Bi}_{\mathrm{a}}$. (b) The temperature variations of a rectangular fin with various $\mathrm{Bi}_{\mathrm{i}}$. 


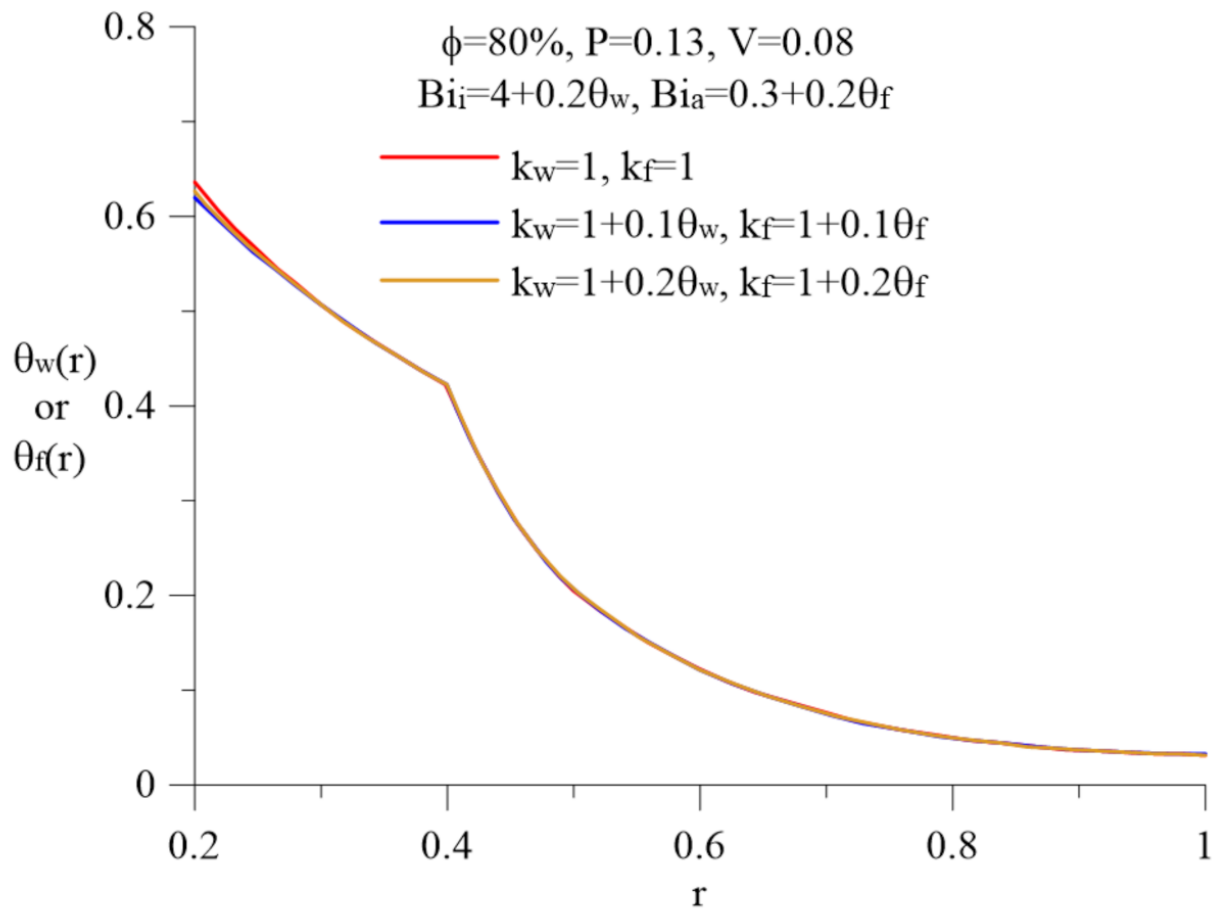

(a)

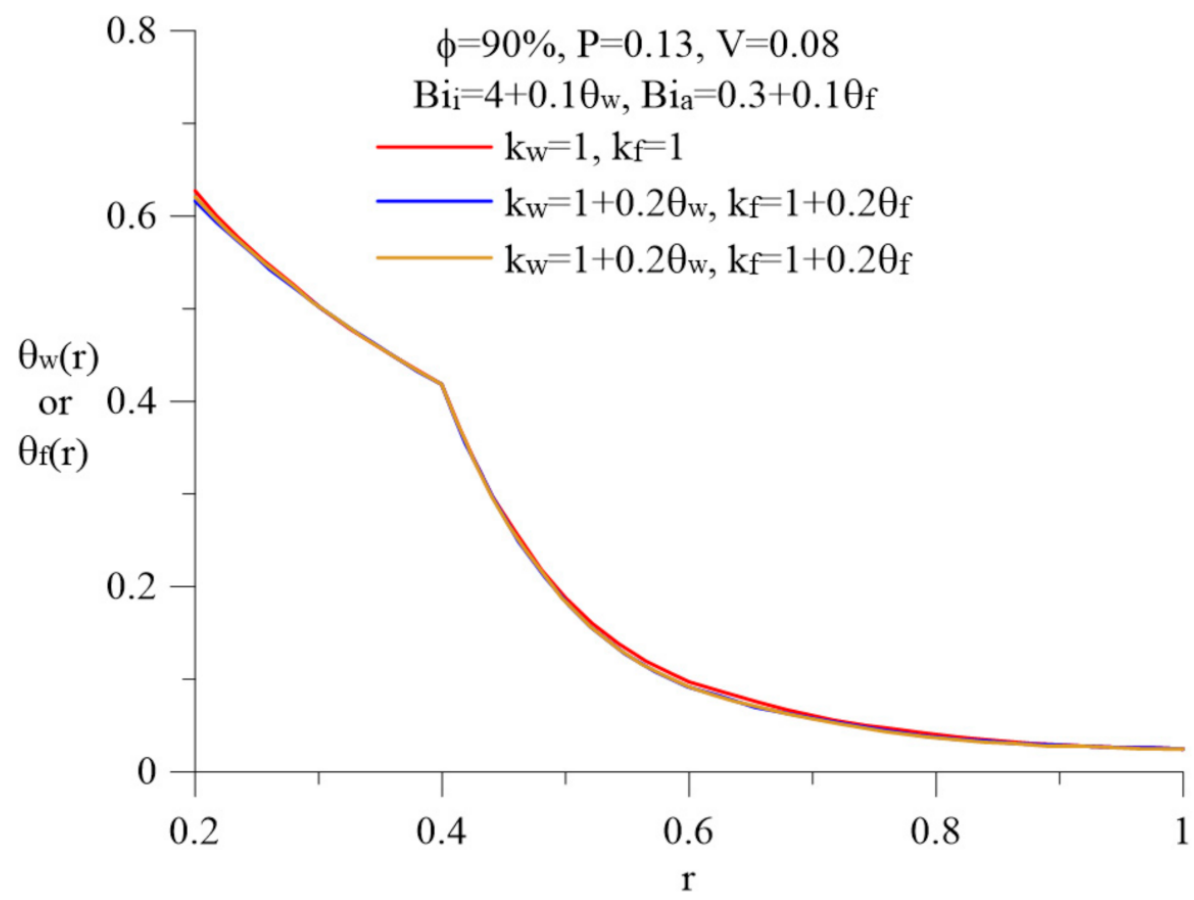

(b)

Figure 4. (a) The temperature variations of a rectangular fin with various $k_{w}$ and $k_{f}$. (b) The temperature variations of a rectangular fin with various $\mathrm{k}_{\mathrm{w}}$ and $\mathrm{k}_{\mathrm{f}}$.

The numerical solutions using various $\operatorname{Bi}_{\mathrm{a}}\left(\theta_{\mathrm{w}}\right)$ are examined first, and the following system parameters are used for the computations: $\mathrm{V}=0.08, \phi=0.85, \mathrm{Bi}_{\mathrm{a}}=0.3,0.3+0.1 \theta_{\mathrm{f}}$ and $0.3+0.2 \theta_{\mathrm{f}}, \mathrm{k}_{\mathrm{w}}=1+0.1 \theta_{\mathrm{w}}, \mathrm{k}_{\mathrm{f}}=1+0.1 \theta_{\mathrm{f}}$ and $\mathrm{Bi}_{\mathrm{i}}=4+0.2 \theta_{\mathrm{w}}$. Figure 3a illustrates the temperature variations in $r$ direction with various $\mathrm{Bi}_{\mathrm{a}}$. As $\mathrm{Bi}_{\mathrm{a}}$ increases from 0.3 to $0.3+0.1 \theta_{\mathrm{f}}$ and then to $0.3+0.2 \theta_{\mathrm{f}}$, the figure shows that there is a discrepancy among the temperature distributions for the linear and non-linear $\mathrm{Bi}_{\mathrm{a}} \mathrm{s}$, and the gap increases as the slope of the non-linear term increases. As a result, the gaps among the optimal fin shapes 
with different $\mathrm{Bi}_{\mathrm{a}}$ will be obvious. It indicates that $\mathrm{Bi}_{\mathrm{a}}$ significantly affects the optimal shape under the condition of $\mathrm{Bi}_{\mathrm{a}}\left(\theta_{\mathrm{w}}\right)$.

Figure $3 \mathrm{~b}$ illustrates the temperature distribution in $\mathrm{r}$ direction. When $\mathrm{Bi}_{\mathrm{i}}$ are increased from 4 to $4+0.1 \theta_{\mathrm{w}}$ and then to $4+0.2 \theta_{\mathrm{W}}$, the temperature differences among the linear and non-linear $\mathrm{Bi}_{\mathrm{i}} \mathrm{s}$ is insignificant, this implies that $\mathrm{Bi}_{\mathrm{i}}$ does not significantly affect the optimal shape of fin under the condition of $\mathrm{Bi}_{\mathrm{i}}\left(\theta_{\mathrm{f}}\right)$.

The numerical solutions using different $\mathrm{k}_{\mathrm{w}}$ and $\mathrm{k}_{\mathrm{f}}$ are examined next. Figure $4 \mathrm{a}$ represents the temperature distributions in $r$ direction with various $\mathrm{k}_{\mathrm{w}}$ and $\mathrm{k}_{\mathrm{f}}$. When $\mathrm{k}_{\mathrm{w}}$ are considered as 1.0, $1.0+0.1 \theta_{\mathrm{w}}$ and $1.0+0.2 \theta_{\mathrm{w}}$ and $\mathrm{k}_{\mathrm{f}}$ are considered as 1.0, $1.0+0.1 \theta_{\mathrm{f}}$ and $1.0+0.2 \theta_{\mathrm{f}}$, small temperature gaps among the constant and temperature-dependent thermal conductivities can be observed near the wall, but in the remaining region they are the same. Next, the temperature distribution in $\mathrm{r}$ direction with $\mathrm{Bi}_{\mathrm{i}}=4+0.1 \theta_{\mathrm{w}}$, $\mathrm{Bi}_{\mathrm{a}}=0.3+0.1 \theta_{\mathrm{f}}$ and $\phi=0.9$ is given in Figure $4 \mathrm{~b}$. The gaps of the temperature distributions among the linear and non-linear thermal conductivities are also small. Therefore, the gaps among optimal fin shapes using different values of $\mathrm{k}_{\mathrm{w}}$ and $\mathrm{k}_{\mathrm{f}}$ will also be found small.

A rectangular fin is considered as the initial design of this work, as shown in Figure 2a. First, the optimal shape of the fin is to be designed by assigning $\Phi=0.3$, the system variables of fin illustrated in Table 1 are used for the computations. The objective function may not converge to the given constraint with the value of desired $\Phi$, but the optimal shape that yields the best computed fin efficiency can be estimated. In this study, the stopping condition is chosen as $\left|\mathrm{J}^{\mathrm{n}}-\mathrm{J}^{\mathrm{n}-1}\right| \leq 10^{-8}$. Table 1 summarizes the results of optimal designs and illustrates that the optimal fin has the highest computed fin efficiency among the examined fins with the identical computational parameters. The interface radii between the wet-dry fin domains $r_{w d}$ are summarized in Table 2. It shows that the optimal fin has the largest value of $r_{w d}$ and, therefore, the highest value of $\eta$.

For the purpose of clearly illustrating the optimum shape with system parameters of $\mathrm{V}=0.08, \phi=0.85, \mathrm{k}_{\mathrm{w}}=1+0.1 \theta_{\mathrm{w}}, \mathrm{k}_{\mathrm{f}}=1+0.1 \theta_{\mathrm{f}}, \mathrm{Bi}_{\mathrm{i}}=4+0.1 \theta_{\mathrm{w}}$ and $\mathrm{Bi}_{\mathrm{a}}=0.3+0.1 \theta_{\mathrm{f}}$, a quarter of an optimal annular fin is given in Figure $5 \mathrm{a}$, while Figure $5 \mathrm{~b}$ illustrates a complete shape of an optimal fin. The thickness of the fin base $\delta\left(r_{o}\right)$ increases from 0.030 for the initial rectangular fin to 0.161 for the optimum fin, and $\eta$ also increases from 0.1365 to 0.2537 . It is clear from Figures 2 and 5 that the fin tip is very sharp and the gap between the initial and optimal fin shapes is obvious.

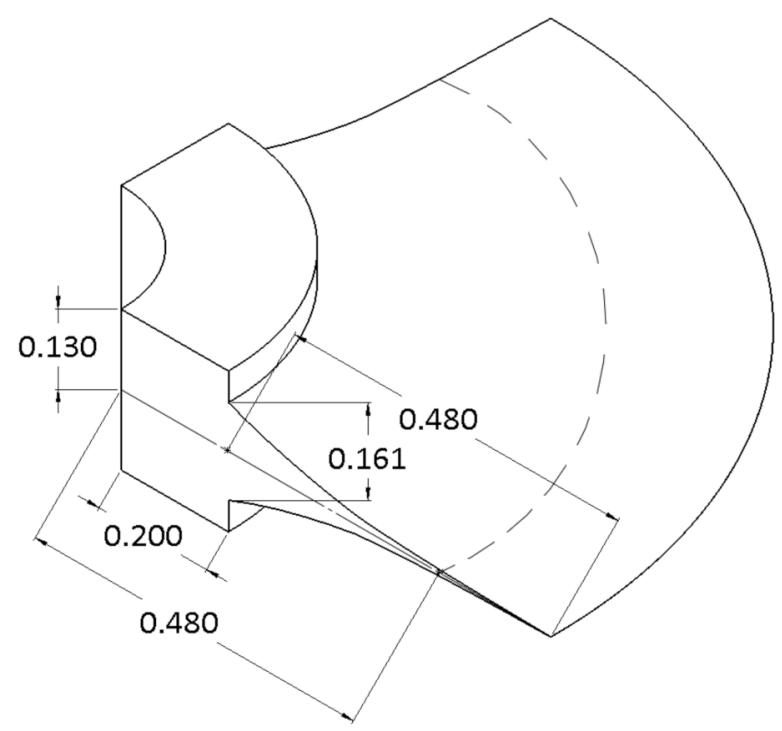

(a)

Figure 5. Cont. 


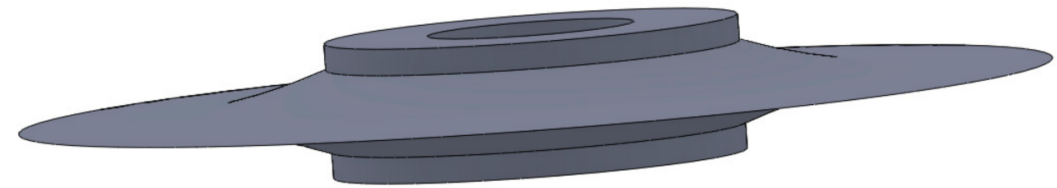

(b)

Figure 5. (a) A quarter of estimated optimal fin. (b) A complete optimum shape annular fin of Figure 5a.

The influence of $\mathrm{Bi}_{\mathrm{a}}$ on the estimated optimum fin shape and $\eta$ is investigated next. Figure 6 a plots the comparisons of $\eta$ for the optimum fin and five commonly seen fins with different $\mathrm{Bi}_{\mathrm{a}}$. Figure $6 \mathrm{a}$ indicates that $\eta$ decreases when $\mathrm{Bi}_{\mathrm{a}}$ increases. The corresponding optimal fin shapes with $\mathrm{Bi}_{\mathrm{a}}=0.3$ and $0.3+0.1 \theta_{\mathrm{f}}$ are given in Figure $6 \mathrm{~b}$. It is seen from the figure that the gaps between the optimum fin shapes with different $\mathrm{Bi}_{\mathrm{a}}$ values near the wall region are obvious.

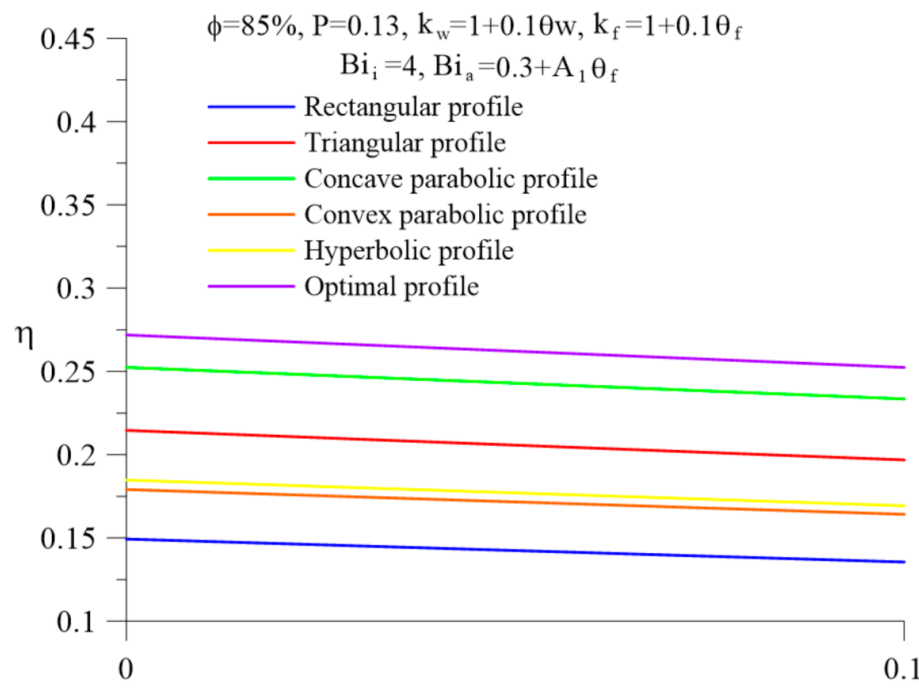

$\mathrm{A}_{1}$

(a)

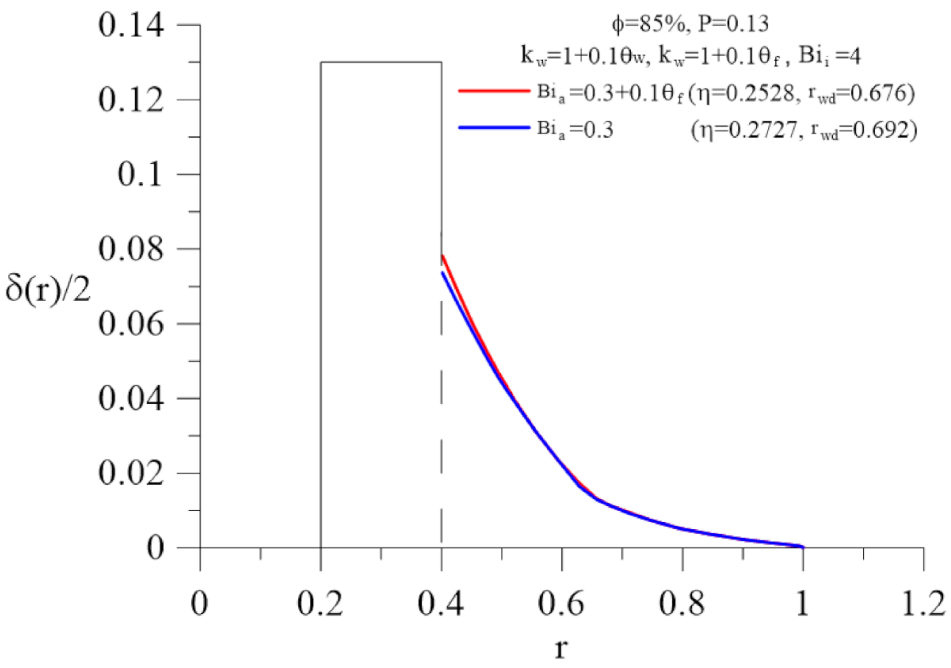

(b)

Figure 6. (a) The comparisons of $\eta$ for different types of partially wet annular fins.with various $\mathrm{Bi}_{\mathrm{a}}$. (b) The estimated optimum fins with various $\mathrm{Bi}_{\mathrm{a}}$.

The comparisons of $\eta$ with different $\mathrm{Bi}_{\mathrm{i}}$ are illustrated in Figure $7 \mathrm{a}$. It indicates that the optimal fin has the highest computed fin efficiency among all fins under the same working 
condition. However, $\eta$ is almost the same as $B i_{i}$ is increased. The estimated optimal fin shapes are plotted in Figure, it indicates that both optimal fin shapes are identical, and it implies that the values of $\mathrm{Bi}_{\mathrm{i}}$ cannot affect the optimum fin shape.

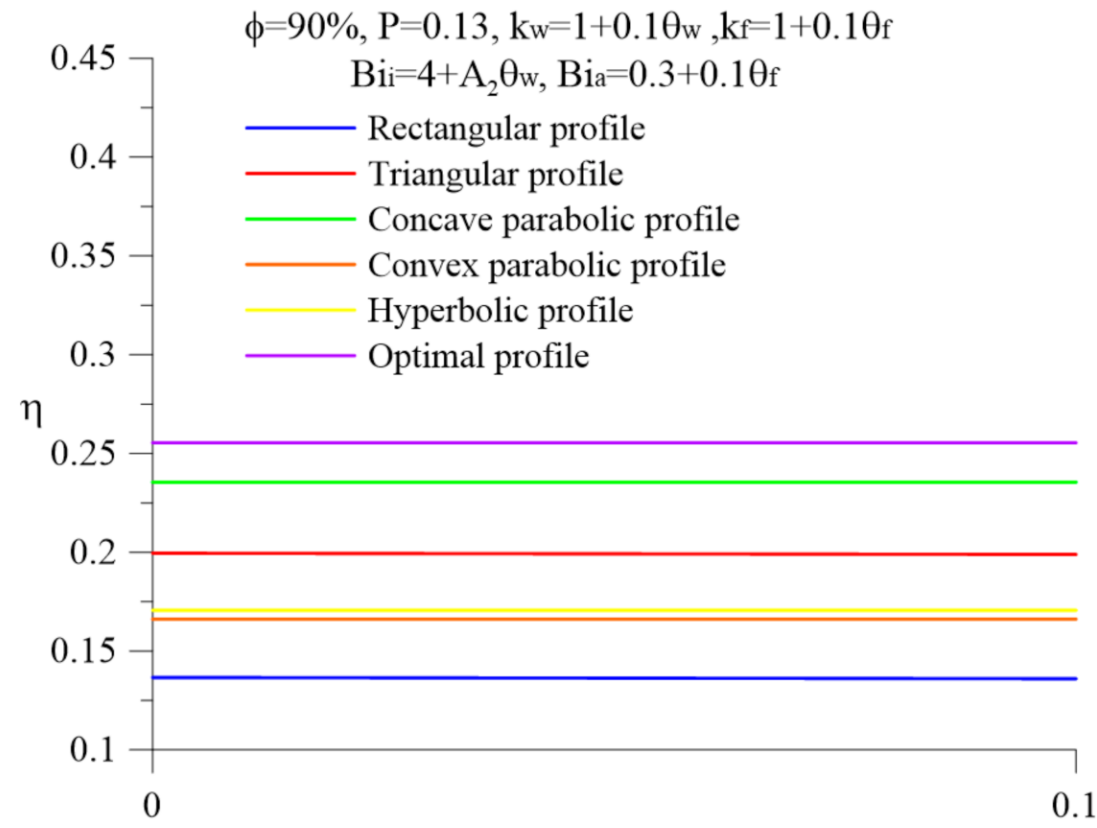

$\mathrm{A}_{2}$

(a)

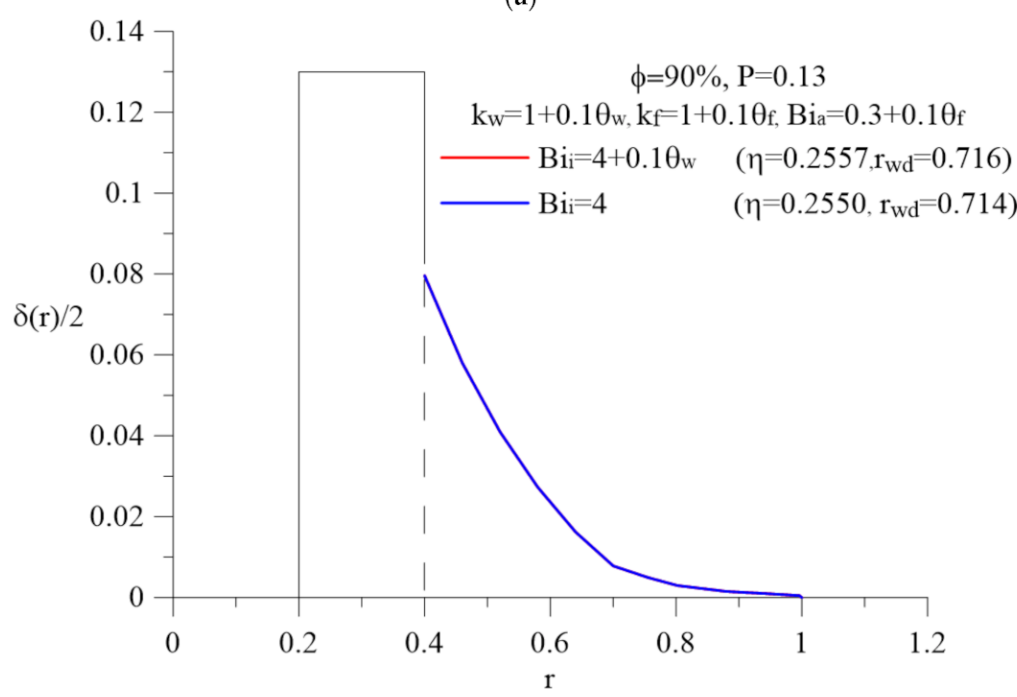

(b)

Figure 7. (a) The comparisons of $\eta$ for different types of fins with various $B i_{i}$. (b) The estimated optimum shapes of fins with various $\mathrm{Bi}_{\mathrm{i}}$.

The comparisons of $\eta$ under the working conditions $\mathrm{Bi}_{\mathrm{i}}=4, \mathrm{Bi}_{\mathrm{a}}=0.3$ and $\mathrm{V}=0.08$ are illustrated in Figure 8a. Again, the optimum fin has the highest $\eta$ among all fins. In addition, $\eta$ remains almost unchanged as the thermal conductivities varied. Figure $8 b$ indicates the optimum fin shapes in Figure 8a. The difference between the optimal fin shapes with different $\mathrm{k}_{\mathrm{w}}$ and $\mathrm{k}_{\mathrm{f}}$ is small for the same reason elaborated previously. 


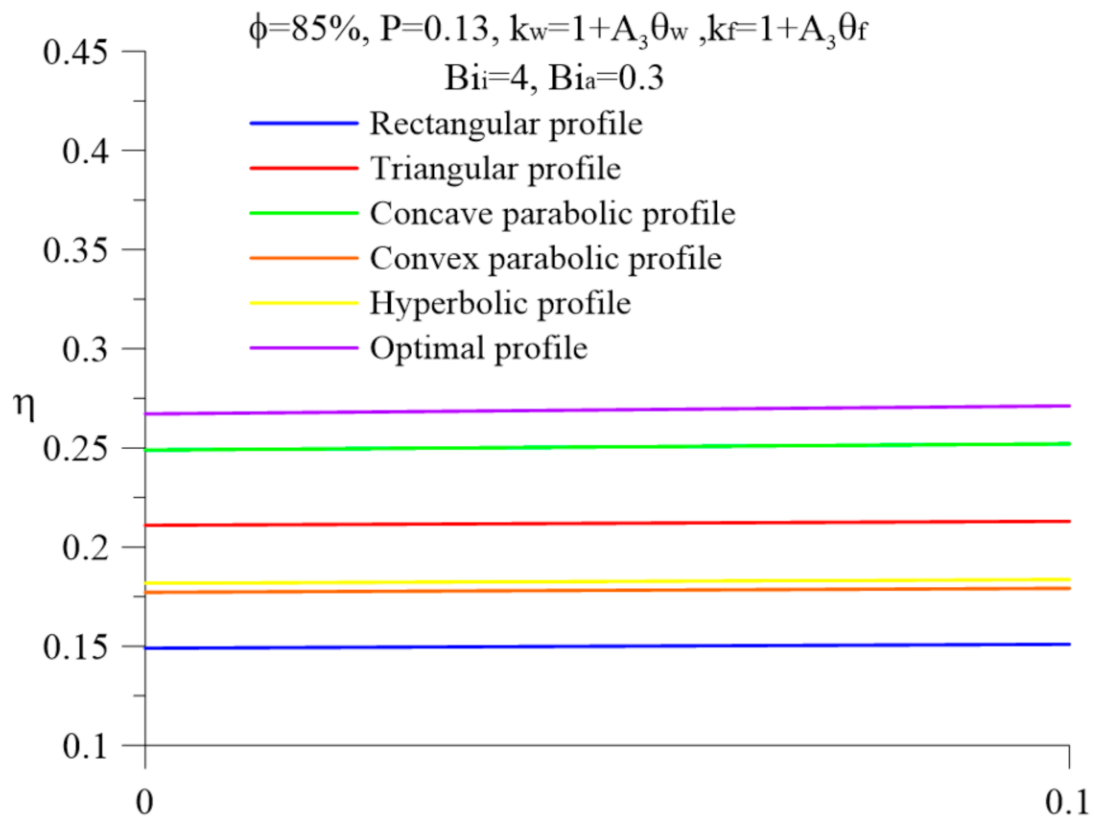

$\mathrm{A}_{3}$

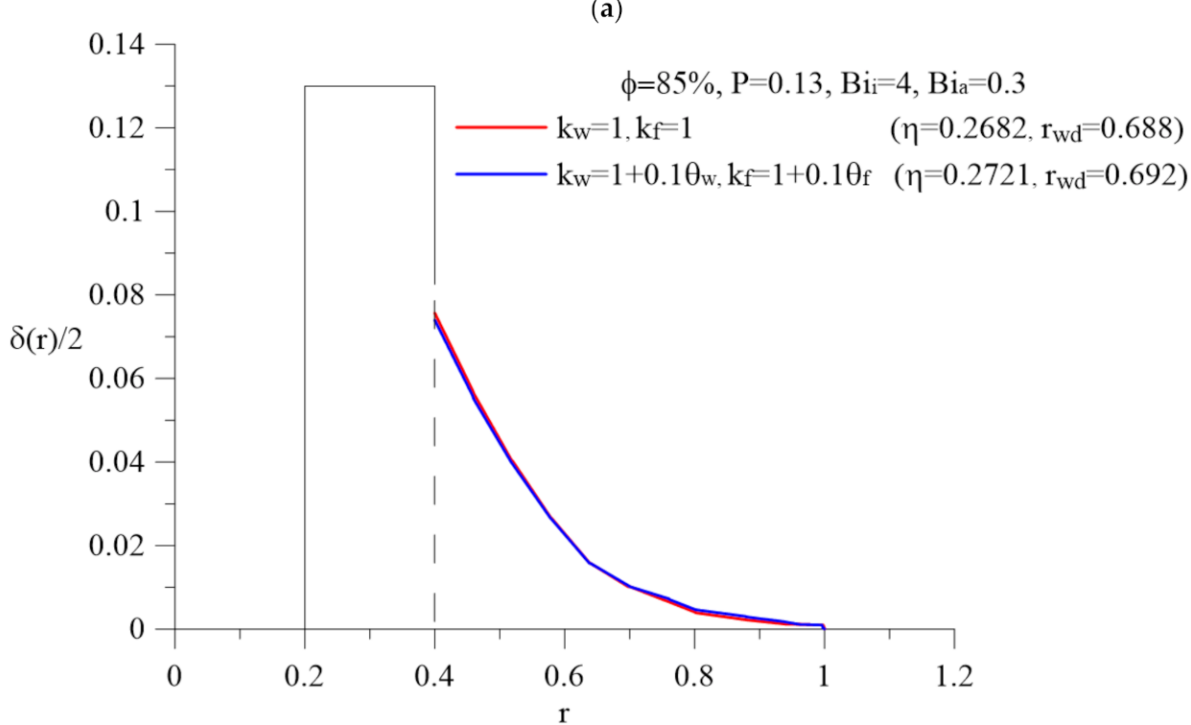

(b)

Figure 8. (a) The comparisons of $\eta$ for different types of fins with various $k_{w}$ and $k_{f}$. (b) The estimated optimal shapes fins with various $\mathrm{k}_{\mathrm{w}}$ and $\mathrm{k}_{\mathrm{f}}$.

Figure 9a,b compares the optimal fin shapes with five commonly seen fins under different system parameters. They indicate that the optimum annular fin has the largest $\eta$ and $r_{w d}$ among all of the fins. It is also noticed that the tip of optimal fin is always very sharp, that is because that thinner and thinner fin thickness along r-direction can procedure higher temperature on fin surface, and as a result, higher computed fin efficiency. 


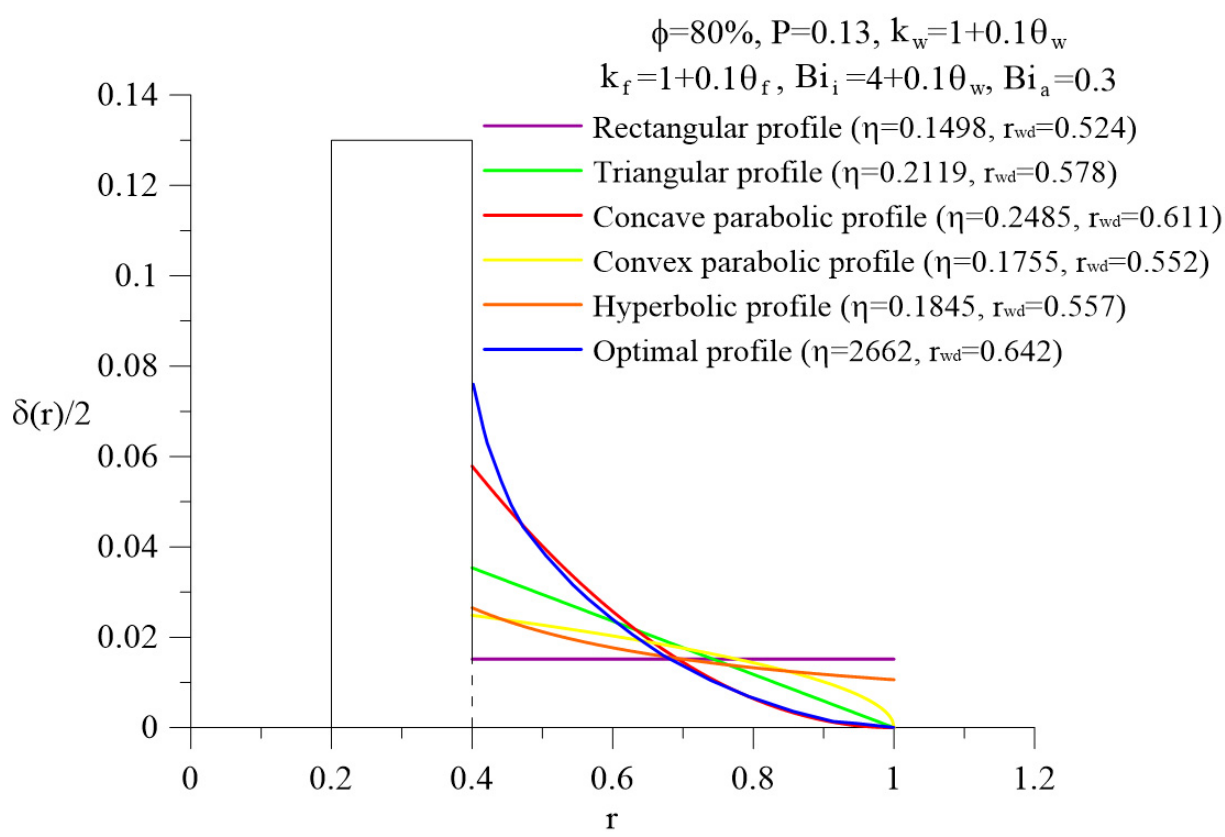

(a)

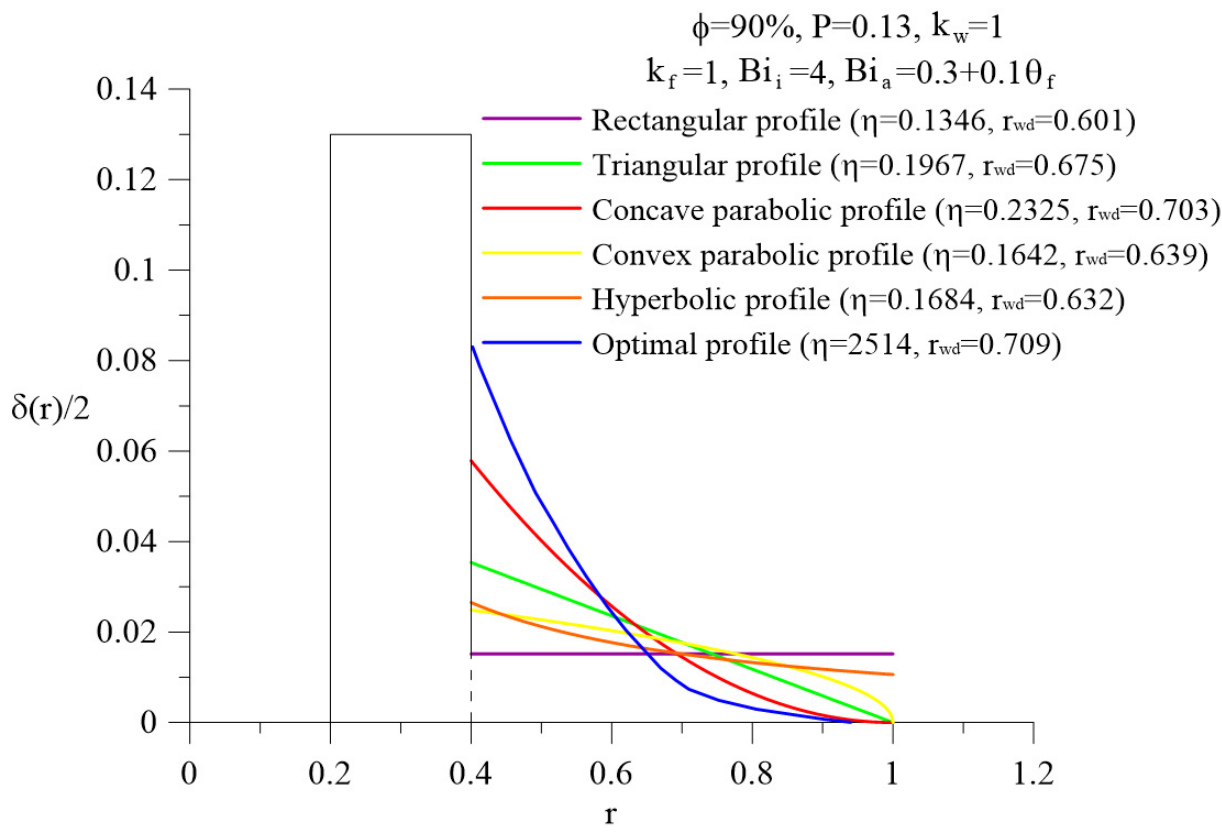

(b)

Figure 9. (a) The comparisons of fin shapes for different types of partially wet annular fins. (b) The comparisons of fin shapes for different types of partially wet annular fins.

\section{Conclusions}

An iterative regularization algorithm using CGM is investigated successfully in the present non-linear design problem to estimate the optimum shape of a partially wet annular fin with temperature-dependent thermal conductivities and Biot number. The relative humidity is varied from 0.8 to 0.85 and then to 0.9 in all designed cases to yield partially wet conditions. Many cases involving various thermal conductivities and Biot numbers are examined, the optimum fin with the highest $\eta$ is found among all fins under identical computational conditions. Moreover, the speed of convergence is also very fast since only $11 \mathrm{~s}$ of CPU time is needed for a maximum of 811 iterations on a CPU $2.8 \mathrm{GHz}$ desktop computer. The computational results indicated that $\mathrm{Bi}_{\mathrm{a}}$ has a significant influence on the 
estimated optimal fin shape and its $\eta$; in addition, when $B \mathrm{i}_{\mathrm{i}}, \mathrm{k}_{\mathrm{w}}$ and $\mathrm{k}_{\mathrm{f}}$ varied, the optimal fin shapes remained approximately identical. It is also found that $\eta$ and $r_{w d}$ increase as $\phi$, $\mathrm{k}_{\mathrm{w}}, \mathrm{k}_{\mathrm{f}}$, and $\mathrm{Bi}_{\mathrm{i}}$ increase and $\mathrm{Bi}_{\mathrm{a}}$ decreases. Due to the fact that the computational time for the present optimal fin shape design problem is very short, the duration of the fin design process can be shortened and the efficiency of the fin design process can be improved. Since the numerical design process for this optimum shape design analysis is completed, the experimental comparison will be conducted in the future for validation.

Author Contributions: Conceptualization, C.-H.H.; methodology, C.-H.H.; software, Y.-L.C.; validation, C.-H.H. and Y.-L.C.; formal analysis, C.-H.H. and Y.-L.C.; investigation, C.-H.H. and Y.-L.C.; writing-original draft preparation, C.-H.H. and Y.-L.C.; writing-review and editing, C.-H.H.; visualization, Y.-L.C.; supervision, C.-H.H.; project administration, C.-H.H.; funding acquisition, C.-H.H. All authors have read and agreed to the published version of the manuscript.

Funding: This research was funded by the Ministry of Science and Technology, Taiwan, grant number MOST-109-2221-E-006-048-MY3.

Institutional Review Board Statement: Not applicable.

Informed Consent Statement: Not applicable.

Data Availability Statement: Not applicable.

Conflicts of Interest: The authors declare no conflict of interest.

\section{Abbreviations}

$\overline{\mathrm{A}}(\overline{\mathrm{r}})$
$\operatorname{Bi}_{\mathrm{a}}\left(\theta_{\mathrm{f}}\right), \mathrm{Bi}_{\mathrm{i}}\left(\theta_{\mathrm{w}}\right)$
$\overline{\mathrm{h}}_{\mathrm{o}}\left(\overline{\mathrm{T}}_{\mathrm{f}}\right), \overline{\mathrm{h}}_{\mathrm{b}}\left(\overline{\mathrm{T}}_{\mathrm{w}}\right)$
$\overline{\mathrm{k}}_{\mathrm{w}}\left(\overline{\mathrm{T}}_{\mathrm{w}}\right), \overline{\mathrm{k}}_{\mathrm{f}}\left(\overline{\mathrm{T}}_{\mathrm{f}}\right)$
$\mathrm{k}_{\mathrm{w}}\left(\theta_{\mathrm{w}}\right), \mathrm{k}_{\mathrm{f}}\left(\theta_{\mathrm{f}}\right)$
$\overline{\mathrm{h}}_{\mathrm{d}}$
$\overline{\mathrm{h}}_{\mathrm{fg}}$
$\mathrm{C}_{\mathrm{p}, \mathrm{a}}$
$\mathrm{J}$
$\mathrm{J}^{\prime}$
$\mathrm{L}_{\mathrm{e}}$
$\mathrm{p}^{\mathrm{n}}$
$\overline{\mathrm{P}}^{\mathrm{p}}$
$\mathrm{P}_{\mathrm{r}}$
$\mathrm{q}$
$\mathrm{Q}$
$\overline{\mathrm{r}}_{\mathrm{b}}, \overline{\mathrm{r}}_{\mathrm{o}}$
$\overline{\mathrm{r}}_{\mathrm{wd}}$
$\overline{\mathrm{r}}_{\mathrm{t}}$
$\overline{\mathrm{S}}(\overline{\mathrm{r}})$
$\mathrm{S}_{\mathrm{c}}$
$\overline{\mathrm{T}}_{\mathrm{w}}, \overline{\mathrm{T}}_{\mathrm{f}}, \overline{\mathrm{w}}_{\mathrm{f}}, \mathrm{d}$
$\overline{\mathrm{T}}_{\mathrm{f}}, \overline{\mathrm{T}}_{\mathrm{c}}, \overline{\mathrm{T}}_{\mathrm{h}}$
$\mathrm{u}($.
$\mathrm{v}(\delta)$
$\mathrm{V}$

fin cross-sectional area $\left(\mathrm{m}^{2}\right)$

Biot number

convective heat transfer coefficient $\left(\mathrm{Wm}^{-2} \mathrm{~K}^{-1}\right)$ temperature-dependent thermal conductivity $\left(\mathrm{Wm}^{-1} \mathrm{~K}^{-1}\right)$ dimensionless temperature-dependent thermal conductivity mass transfer coefficient $\left(\mathrm{kgm}^{-2} \mathrm{~s}^{-1}\right)$

latent heat of condensation of moisture $\left(\mathrm{Jkg}^{-1}\right)$

constant pressure specific heat for moist air $\left(\mathrm{Jkg}^{-1} \mathrm{~K}^{-1}\right)$

cost function defined by Equation (3)

gradient of the cost function defined by Equation (15)

Lewis number

search direction

half fin pitch (m)

Prandtl number

actual heat transfer rate of fin

ideal heat transfer rate of fin

inner and external tube radius (m)

interfacial radius between wet and dry fin domains $(\mathrm{m})$

external fin radius $(\mathrm{m})$

fin perimeter $(\mathrm{m})$

Schmidt number

tube wall, wet fin and dry fin surfaces temperatures $(\mathrm{K})$

fin, refrigerant and air temperatures $(\mathrm{K})$

unit step function

estimated fin volume

specified fin volume 


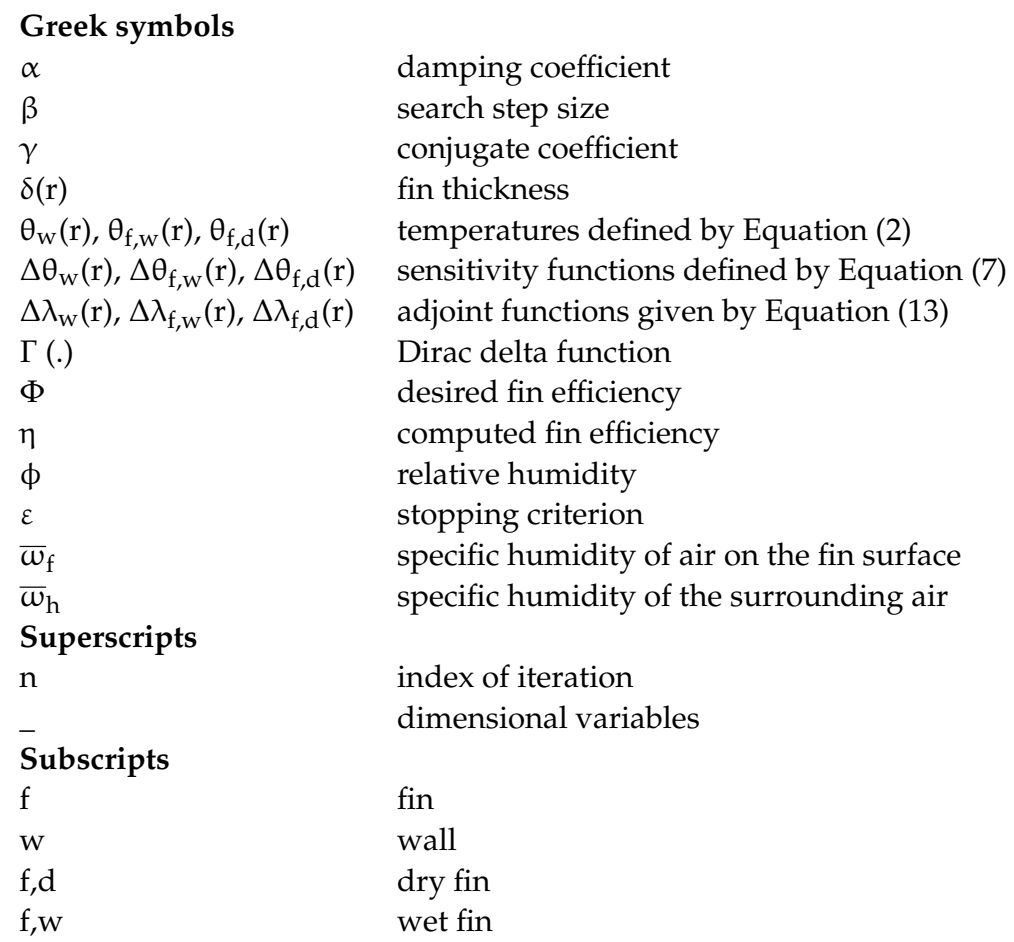

\section{References}

1. Charters, W.W.S.; Theerakulpisut, S. Efficiency equations for constant thickness annular fins. Int. Commun. Heat Mass Transf. 1989, 16, 547-558. [CrossRef]

2. Mokheimer, E.M.A. Performance of annular fins with different profiles subject to variable heat transfer coefficient. Int. J. Heat Mass Transf. 2002, 45, 3631-3642. [CrossRef]

3. Iborra, A.A.; Campo, A. Approximate analytic temperature distribution and efficiency for annular fins of uniform thickness. Int. J. Therm. Sci. 2009, 48, 773-780. [CrossRef]

4. McQuiston, F.C. Fin efficiency with combined heat and mass transfer. ASHRAE Trans. 1975, 81, 350-355.

5. Toner, M.; Kilic, A.; Onat, K. Comparison of rectangular and triangular fins when condensation occurs. Heat Mass Transf. 1983, 17, 65-72. [CrossRef]

6. Schmidt, E. Die Warmeubertragung durch Rippen. Z. VDI 1926, 70, 885-947.

7. Kern, D.Q.; Kraus, A.D. Extended Surface Heat Transfer; MacGraw-Hill: New York, NY, USA, 1972.

8. Jang, Y.J.; Choi, D.J.; Kim, S.; Hyun, M.T.; Lee, Y.G. Enhancement of condensation heat transfer rate of the air-steam mixture on a passive condenser system using annular fins. Energies 2017, 10, 1777. [CrossRef]

9. Xu, Z.; Guo, Y.; Yang, H.; Mao, H.; Yu, Z.; Li, R. One convenient method to calculate performance and optimize configuration for annular radiator using heat transfer unit simulation. Energies 2020, 13, 271. [CrossRef]

10. Wu, G.; Bong, T.Y. Overall efficiency of a straight fin with combined heat and mass transfer. Am. Soc. Heat. Refrig. Air-Cond. Eng. Trans. 1994, 100, 367-374.

11. Naphon, P. Study on the heat transfer characteristics of the annular fin under dry-surface, partially wet-surface, and fully wet-surface conditions. Int. Commun. Heat Mass Transf. 2006, 33, 112-121. [CrossRef]

12. Pirompugd, W.; Wang, C.C.; Wongwises, S. Finite circular fin method for heat and mass transfer characteristics for plain fin-and-tube heat exchangers under fully and partially wet surface conditions. Int. J. Heat Mass Transf. 2007, 50, 552-565. [CrossRef]

13. Rosario, L.; Rahman, M.M. Analysis of heat transfer in a partially wet radial fin assembly during dehumidification. Int. J. Heat Fluid Flow 1999, 20, 642-648. [CrossRef]

14. Kang, H.S. Optimization of a rectangular profile annular fin based on fixed fin height. J. Mech. Sci. Technol. 2009, 23, 3124-3131. [CrossRef]

15. Brown, A. Optimum dimensions of uniform annular fins. Int. J. Heat Mass Transf. 1964, 8, 655-662. [CrossRef]

16. Huang, C.H.; Hsiao, J.H. A non-linear fin design problem in determining the optimum shape of spine and longitudinal fins. Commun. Numer. Methods Eng. 2003, 19, 111-124. [CrossRef]

17. Alifanov, O.M. Solution of an inverse problem of heat conduction by iteration methods. J. Eng. Phys. 1974, 26, 471-476. [CrossRef]

18. Huang, C.H.; Chung, Y.L. Optimal Design of Annular Fin Shapes with Temperature-Dependent Properties. AIAA J. Thermophys. Heat Transfer. 2018, 32, 18-26. [CrossRef]

19. Huang, C.H.; Chung, Y.L. A Nonlinear Fin Design Problem in Determining the Optimum Shapes of Fully Wet Annular Fins. Appl. Therm. Eng. 2016, 103, 195-204. [CrossRef] 
20. Huang, C.H.; Chung, Y.L. An inverse problem in determining the optimum shapes for partially wet annular fins based on efficiency maximization. Int. J. Heat Mass Transf. 2015, 90, 364-375. [CrossRef]

21. Sharqawy, M.H.; Zubair, S.M. Efficiency and optimization of an annular fin with combined heat and mass transfer-An analytical solution. Int. J. Refrig. 2007, 30, 751-757. [CrossRef]

22. Threlkeld, J.L. Thermal Environmental Engineering, 2nd ed.; Prentice-Hall, Inc.: Englewood Cliffs, NJ, USA, 1970. 